\title{
LOCAL ASYMPTOTIC MIXED NORMALITY OF APPROXIMATE MAXIMUM LIKELIHOOD ESTIMATOR OF DRIFT PARAMETERS IN DIFFUSION MODEL
}

\author{
Snježana Lubura Strunjak And Miljenko Huzak \\ University of Zagreb, Croatia
}

\begin{abstract}
We assume that the diffusion $X$ satisfies a stochastic differential equation of the form: $d X_{t}=\mu\left(X_{t}, \theta\right) d t+\sigma_{0} \nu\left(X_{t}\right) d W_{t}$, with unknown drift parameter $\theta$ and known diffusion coefficient parameter $\sigma_{0}$. We prove that approximate maximum likelihood estimator of drift parameter $\bar{\theta}_{n}$ obtained from discrete observations $\left(X_{i \Delta_{n}}, 0 \leq i \leq n\right)$ along fixed time interval $[0, T]$, and when $\Delta_{n}=\frac{T}{n}$ tends to zero, is locally asymptotic mixed normal, with covariance matrix which depends on MLE $\hat{\theta}$ obtained from continuous observations $\left(X_{t}, 0 \leq t \leq T\right)$ along fixed time interval $[0, T]$, and on path $\left(X_{t}, 0 \leq t \leq T\right)$.
\end{abstract}

\section{INTRODUCTION}

Let $W=\left(W_{t}, t \geq 0\right)$ be a one-dimensional standard Brownian motion defined on filtered probability space $\left(\Omega, \mathcal{F},\left(\mathcal{F}_{t}\right)_{t \geq 0}, \mathbb{P}\right)$. We suppose that filtration $\left(\mathcal{F}_{t}\right)_{t \geq 0}$ is the smallest one such that satisfies the usual conditions and to which $W$ is adapted. Let $X=\left(X_{t}, t \geq 0\right)$ be a one-dimensional diffusion which satisfies Itô's stochastic differential equation (SDE) of the form (for details see [19])

$$
d X_{t}=\mu\left(X_{t}, \theta\right) d t+\sigma_{0} \nu\left(X_{t}\right) d W_{t}, \quad X_{0}=x_{0}, \quad t \geq 0,
$$

2010 Mathematics Subject Classification. 62M05, 62F12, 60J60.

Key words and phrases. Asymptotic mixed normality, diffusion processes, discrete observation, parameter estimation.

This work has been fully supported by Croatian Science Foundation under the project 3526 . 
where $\nu$ and $\mu$ are real functions and $x_{0}$ is a given deterministic initial value of $X$. For example, if both functions $\nu$ and $\mu(\cdot, \theta)$ are locally Lipschitz, then diffusion given by (1.1) has strong solution, see [20, Theorem V.12.1].

Let $\theta_{0}$ and $\sigma_{0}$ be true values of drift parameter and diffusion coefficient parameter respectively. We assume that $\sigma_{0}>0$ is known. Let $T>0$ be a fixed real number and $0=: t_{0}<t_{1}<\cdots<t_{n}:=T, n \in \mathbb{N}$ be a deterministic subdivision of segment $[0, T]$ such that $t_{i}=i \Delta_{n}, i=0, \ldots, n, \Delta_{n}=\frac{T}{n}$. Given a discrete observation $\left(X_{t_{i}}, 0 \leq i \leq n\right)$ of the trajectory $\left(X_{t}, t \in[0, T]\right)$, the problem is to estimate the unknown drift parameter $\theta$ of $X$. We assume that $\theta$ belongs to the parameter space $\Theta$ which is a relatively compact, open and convex set in Euclidean space $\mathbb{R}^{d}$.

The maximum likelihood estimator (MLE) of drift parameters based on continuous observation $\left(X_{t}, t \in[0, T]\right)$ has good properties, such as consistency and asymptotic efficiency when $T$ goes to infinity in case of ergodic diffusions (see $[3,16]$ ). The same holds for MLE based on discrete observations (see [4]) but in most cases it can not be explicitly calculated. Hence, other methods of estimations have to be considered. In this paper we use method based on a Gaussian approximation of the transition density (see [14]) to construct contrast function used for getting approximate maximum likelihood estimator (AMLE) of drift parameters based on discrete observations. We consider the simplest case based on approximation of diffusion $X$ by a solution to difference equation obtained from (1.1) by Euler approximation of Riemann and Itô integrals (see $[9,15]$ ). In such a way obtained AMLE is consistent and asymptotically normal in case of ergodic diffusions when $n \Delta_{n}$ goes to infinity $(T \rightarrow+\infty)$ such that $n \Delta_{n}^{3}$ goes to zero (see [6]). In case when $T$ is fixed and $\Delta_{n}$ goes to zero it was proved that measurable AMLE exists, and it converges to MLE based on continuous observations over $[0, T]$ with rate $\sqrt{\Delta_{n}}$ in probability (see [15] for linear case, and [10] for general case).

The existence, measurability and some asymptotic properties of the AMLE are proved in [15], under assumption that observations can be taken only up to some maximal observational time $T$, when $\Delta_{n}$ tends to zero and under the assumption that the diffusion satisfies a linear SDE with completely determined and constant diffusion coefficient function. The similar results are proved in [10] in more general case (without assumption on linearity of SDE).

In this paper we prove that the difference between AMLE and MLE based on continuous observations over fixed observational time interval $[0, T]$, normed with $\sqrt{\Delta_{n}}$ is asymptotically mixed normal when $\Delta_{n}$ goes to zero, and that covariance matrix of the limit random vector depends on MLE and the continuous trajectory. This result can be applied for estimations of AMLE's standard errors, and hence asymptotic confidence intervals for drift parameters in ergodic diffusions case, which include a correction due to discretization, for example, in a sense of Example 6.3 from Sect. 6. 
The paper is organized in the following way. In the next section we introduce notation and definitions we need. The main results are presented in Sect. 4, followed by proofs in Sect. 5. In the last section we will present simulation study throughout two examples.

\section{Preliminaries}

We denote by $\langle\cdot, \cdot\rangle$ the scalar product in the $d$-dimensional Euclidean space $\mathbb{R}^{d}$ and by $\|\cdot\|$ the induced norm. The drift parameter space $\Theta \subseteq \mathbb{R}^{d}$ is relatively compact, which means that its closure $C l(\Theta)$ is a compact set in $\mathbb{R}^{d}$. We will denote by $E$ an open interval such that $E \subseteq \mathbb{R}$. We will say that $U$ is relatively compact in $E$ if $U \subseteq E$ and $C l(U)$ is compact set in $E$. If $(x, \theta) \mapsto f(x, \theta)$ is a real function then we will denote by $D_{\theta}^{m} f$ the $m$-th partial derivatives with respect to $\theta$ of the function $f, m \in \mathbb{N}$, provided that they exist. If $\theta \mapsto f(\theta)$ is a real-valued function defined on an open subset of $\mathbb{R}^{d}$, then we will denote by $D f(\theta), D^{2} f(\theta)$ its first and second derivatives with respect to $\theta$, provided that they exist. Let $K$ be a relatively compact set in $\mathbb{R}^{d}$. We say that a partial derivative $D_{\theta}^{m} f$ of a real function $f: E \times C l(K) \rightarrow \mathbb{R}$ exists on $E \times C l(K)$ if there exists an open set $U^{K} \subseteq \mathbb{R}^{d}$, such that $E \times C l(K) \subseteq E \times U^{K}$, and an extension $\tilde{f}$ of $f$, defined on $E \times U^{K}$, such that $D_{\theta}^{m} \tilde{f}$ exists.

For fixed $T>0$ let $\left(\Omega, \mathcal{F}_{T},\left(\mathcal{F}_{t}\right)_{0 \leq t \leq T}, \mathbb{P}\right)$ be a given filtered probability space. Let $\left(\tilde{\Omega}, \tilde{\mathcal{F}}_{T}, \tilde{\mathbb{F}}=\left(\tilde{\mathcal{F}}_{t}\right)_{0 \leq t \leq T}, \tilde{\mathbb{P}}\right)$ be an extension of $\left(\Omega, \mathcal{F}_{T},\left(\mathcal{F}_{t}\right)_{0 \leq t \leq T}, \mathbb{P}\right)$ (for details see [11]). The extension is called very good if all martingales on the space $\left(\Omega, \mathcal{F}_{T},\left(\mathcal{F}_{t}\right)_{0 \leq t \leq T}, \mathbb{P}\right)$ are also martingales on $\left(\tilde{\Omega}, \tilde{\mathcal{F}}_{T}, \tilde{\mathbb{F}}=\left(\tilde{\mathcal{F}}_{t}\right)_{0 \leq t \leq T}, \tilde{\mathbb{P}}\right)$. Let $A$ be some Polish space. In our case $A$ will be the Skorokhod space $A=D\left([0, T], \mathbb{R}^{d}\right)$ or $d$-dimensional Euclidean space $A=\mathbb{R}^{d}$. Let $\left(Z_{n}\right)$ be a sequence of $A$-valued random vectors, all defined on $\left(\Omega, \mathcal{F}_{T},\left(\mathcal{F}_{t}\right)_{0 \leq t \leq T}, \mathbb{P}\right)$, and let $Z$ be an $A$-valued random vector defined on the extension $\left(\tilde{\Omega}, \tilde{\mathcal{F}}_{T}, \tilde{\mathbb{F}}=\right.$ $\left.\left(\tilde{\mathcal{F}}_{t}\right)_{0 \leq t \leq T}, \tilde{\mathbb{P}}\right)$. We will say that $\left(Z_{n}\right)$ converges stably in law to $Z$, and write $Z_{n} \stackrel{s t}{\Rightarrow} Z$, if

$$
\lim _{n \rightarrow \infty} \mathbb{E}\left[Y f\left(Z_{n}\right)\right]=\tilde{\mathbb{E}}[Y f(Z)],
$$

for all bounded continuous functions $f: A \rightarrow \mathbb{R}$ and all bounded random variable $Y$ on $\left(\Omega, \mathcal{F}_{T},\left(\mathcal{F}_{t}\right)_{0 \leq t \leq T}, \mathbb{P}\right)$. This kind of convergence is introduced by Rényi (see [18]) and studied by Aldous and Eagleson (see [1]). Nice properties of this kind of convergence can also be found in $[12,11]$.

We will say that an $\mathbb{R}^{d}$-valued random vector $Y$ has mixed normal distribution with $\mathcal{F}_{T}$-measurable random covariance matrix $C=\left(C^{j k}\right)_{j, k=1, \ldots, d}$, and we write $Y \sim M N(0, C)$ if

$$
\mathbb{E}\left[e^{i\langle t, Y\rangle} \mid \mathcal{F}_{T}\right]=e^{-\frac{1}{2} \sum_{j, k=1, \ldots, d} t_{j} t_{k} C^{j k}}
$$


(see [1]). If $Y \sim M N(0, C)$, then $Y$ has the same distribution as $\sqrt{C} Z$, where $\sqrt{C}$ is square symmetric root of $C$ and $Z \sim N(0, I)$ is standard normal random vector independent of $\mathcal{F}_{T}$. An $\mathbb{R}^{d}$-valued process $\left(Y_{t}\right)_{0 \leq t \leq T}$ is centered Gaussian process if for all $0 \leq s_{1}<s_{2}<\cdots<s_{k} \leq T$ random matrix $\left(Y_{s_{1}}, \ldots, Y_{s_{k}}\right) \in \mathbb{R}^{d k}$ has multivariate normal distribution and $\mathbb{E}\left[Y_{t}\right]=0, t \in$ $[0, T]$.

For $n \in \mathbb{N}$, let $t_{i}:=i \Delta_{n}, i=0, \ldots, n$ be an equidistant subdivision of segment $[0, T], \Delta_{n}=\frac{T}{n}$. Let $A_{n}^{t}:=\max \left\{j: t_{j} \leq t\right\}$ and $\mathcal{F}_{n, i}:=\mathcal{F}_{t_{i}}, i=$ $0, \ldots, n, n \in \mathbb{N}$. For proving local mixed normality of the AMLE we use the following theorem, which is a version of [11, Theorem 3-2], but with notation adjusted for our article.

TheOREM 2.1. Let $W$ be a one-dimensional Brownian motion on $\left(\Omega, \mathcal{F}_{T}\right.$, $\left.\mathbb{F}=\left(\mathcal{F}_{t}\right)_{0 \leq t \leq T}, \mathbb{P}\right)$, and let $\chi_{i}^{n}$ be $\mathcal{F}_{t_{i}}$-measurable square integrable $\mathbb{R}^{d}$-valued random vectors. Let $C=\left(C^{j k}\right)$ be continuous adapted process defined on $\left(\Omega, \mathcal{F}_{T}, \mathbb{F}=\left(\mathcal{F}_{t}\right)_{0 \leq t \leq T}, \mathbb{P}\right)$ such that $C_{t}$ is positive semidefinite symmetric $d \times d$ matrix for all $t \in[0, T]$. Assume that next conditions hold:

$$
\begin{array}{ll}
\sup _{0 \leq t \leq T}\left\|\sum_{i=1}^{A_{n}^{t}} \mathbb{E}\left[\chi_{i}^{n} \mid \mathcal{F}_{n, i-1}\right]\right\| \stackrel{\mathbb{P}}{\rightarrow} 0, & \\
\sum_{i=1}^{A_{n}^{t}}\left(\mathbb{E}\left[\chi_{i}^{n, j} \chi_{i}^{n, k} \mid \mathcal{F}_{n, i-1}\right]-\mathbb{E}\left[\chi_{i}^{n, j} \mid \mathcal{F}_{n, i-1}\right] \mathbb{E}\left[\chi_{i}^{n, k} \mid \mathcal{F}_{n, i-1}\right]\right) \stackrel{\mathbb{P}}{\rightarrow} C_{t}^{j, k}, \\
\forall t \in[0, T], j, k=1, \ldots, d, & \forall t \in[0, T], \\
\sum_{i=1}^{A_{n}^{t}} \mathbb{E}\left[\chi_{i}^{n}\left(W_{t_{i}}-W_{t_{i-1}}\right) \mid \mathcal{F}_{n, i-1}\right] \stackrel{\mathbb{P}}{\rightarrow} 0, & \forall \epsilon>0, \\
\sum_{i=1}^{n} \mathbb{E}\left[\left\|\chi_{i}^{n}\right\|^{2} 1_{\left\{\left\|\chi_{i}^{n}\right\|>\epsilon\right\}} \mid \mathcal{F}_{n, i-1}\right] \stackrel{\mathbb{P}}{\rightarrow} 0, & \\
\sum_{i=1}^{A_{n}^{t}} \mathbb{E}\left[\chi_{i}^{n}\left(N_{t_{i}}-N_{t_{i-1}}\right) \mid \mathcal{F}_{n, i-1}\right] \stackrel{\mathbb{P}}{\rightarrow} 0, & \forall t \in[0, T],
\end{array}
$$

where $N$ is bounded $\mathcal{F}_{t}$-martingale orthogonal to $W$.

Then we have

$$
\sum_{i=1}^{A_{n}^{t}} \chi_{i}^{n} \stackrel{s t}{\Rightarrow} Y \quad \text { on } D\left([0, T], \mathbb{R}^{d}\right),
$$

where $Y$ is a continuous process defined on a very good filtered extension $\left(\tilde{\Omega}, \tilde{\mathcal{F}}, \tilde{\mathbb{F}}=\left(\tilde{\mathcal{F}}_{t}\right)_{0 \leq t \leq T}, \tilde{\mathbb{P}}\right)$ of $\left(\Omega, \mathcal{F}_{T}, \mathbb{F}=\left(\mathcal{F}_{t}\right)_{0 \leq t \leq T}, \mathbb{P}\right)$ and which, conditionally on $\sigma$-field $\mathcal{F}_{T}$, is centered Gaussian $\mathbb{R}^{d}$-valued process with independent increments satisfying $\tilde{\mathbb{E}}\left[Y_{t}^{j} Y_{t}^{k} \mid \mathcal{F}_{T}\right]=C_{t}^{j k}, t \in[0, T], j, k=1, \ldots, d$. 
Let $\left(\gamma_{n}, n \in \mathbb{N}\right)$ be a sequence of positive numbers and let $Y=\left(Y_{n}, n \in \mathbb{N}\right)$ be a sequence of random vectors defined on some probability space. We will say that $Y$ is of order $O_{\mathbb{P}}\left(\gamma_{n}\right), n \in \mathbb{N}$, and we will write $Y_{n}=O_{\mathbb{P}}\left(\gamma_{n}\right)$, if $\left(\frac{Y_{n}}{\gamma_{n}}, n \in \mathbb{N}\right)$ is bounded in probability, which means that

$$
\lim _{A \rightarrow+\infty} \varlimsup_{n} \mathbb{P}\left\{\gamma_{n}^{-1}\left\|Y_{n}\right\|>A\right\}=0 .
$$

We will denote $L^{1}(\mathbb{P})$-norm by $\|\cdot\|_{1}:=\mathbb{E}[|\cdot|]$ and $L^{2}(\mathbb{P})$-norm by $\|\cdot\|_{2}:=$ $\sqrt{\mathbb{E}\left[(\cdot)^{2}\right]}$.

\section{Estimation Method}

Let us now introduce our AMLE of $d$-dimensional parameter $\theta \in \Theta$. Let $X$ be a strong solution of $\operatorname{SDE}$ (1.1) for true parameter value $\theta_{0} \in \Theta$ and given Brownian motion $W$. If we discretize the SDE over interval $\left[t_{i-1}, t_{i}\right]$ by using the Euler discretization scheme, we get stochastic difference equation of the form:

(3.1) $Z_{t_{i}}-Z_{t_{i-1}}=\mu\left(Z_{t_{i-1}}, \theta\right)\left(t_{i}-t_{i-1}\right)+\sigma_{0} \nu\left(Z_{t_{i-1}}\right)\left(W_{t_{i}}-W_{t_{i-1}}\right), \quad Z_{0}=x_{0}$,

for $1 \leq i \leq n$. If it exists, the solution of (3.1) is a discrete-time process $Z=\left(Z_{t_{0}}, Z_{t_{1}}, \ldots, Z_{t_{n}}\right)$ which is an approximation of $X$ on $[0, T]$. The log likelihood function, shortly LLF, of the process $Z$, up to the constant not depending on the parameter $\theta$ is

$$
-\frac{n}{2} \ln \left(\sigma_{0}^{2}\right)-\frac{1}{2} \sum_{i=1}^{n} \frac{\left(z_{i}-z_{i-1}-\mu\left(z_{i-1}, \theta\right)\left(t_{i}-t_{i-1}\right)\right)^{2}}{\sigma_{0}^{2} \nu^{2}\left(z_{i-1}\right)\left(t_{i}-t_{i-1}\right)} .
$$

The contrast function is obtained from $(3.2)$ by substituting vector $\left(z_{0}, \ldots, z_{n}\right)$ with the discrete observations $\left(X_{t_{0}}, X_{t_{1}}, \ldots, X_{t_{n}}\right)$ of the process $X$ and neglecting the part which does not depend on parameter $\theta$ :

$$
L_{n}(\theta)=\sum_{i=1}^{n}\left(\frac{\left(X_{t_{i}}-X_{t_{i-1}}\right) \mu\left(X_{t_{i-1}}, \theta\right)}{\sigma_{0}^{2} \nu^{2}\left(X_{t_{i-1}}\right)}-\frac{1}{2} \frac{\mu^{2}\left(X_{t_{i-1}}, \theta\right)\left(t_{i}-t_{i-1}\right)}{\sigma_{0}^{2} \nu^{2}\left(X_{t_{i-1}}\right)}\right) .
$$

Here we assumed that $\nu \neq 0$ on the state space of $X$. A point of global maximum $\bar{\theta}_{n}$ of the function (3.3), if it exists, is an estimator of the parameter $\theta$ which we call approximate maximum likelihood estimator, shortly AMLE. Further in the text, if it is written point of maximum, it means point of global maximum.

\section{MAin Results}

We assume that drift parameter space $\Theta$ is a relatively compact, open and convex set in $\mathbb{R}^{d}, d \geq 1$, and state space $E$ is an open interval in $\mathbb{R}$.

Let the following assumptions be satisfied: 
(A1) For all $\theta \in \Theta$, there exists strong solution $X$ of $\operatorname{SDE}$ (1.1) on time interval $[0,+\infty\rangle$. This solution $X$ has continuous paths with values in $E$ and if $X^{\prime}$ is any other solution of SDE (1.1), with the same Brownian motion $W$, then the law of $X^{\prime}$ is identical to the law of $X$.

(A2) For all $\theta \in C l(\Theta)$, function $\mu(\cdot, \theta): E \rightarrow \mathbb{R}$ is continuously differentiable on $E$. Functions $(x, \theta) \mapsto \mu(x, \theta),(x, \theta) \mapsto \frac{\partial^{3}}{\partial x^{2} \partial \theta_{j}} \mu(x, \theta)$, $(x, \theta) \mapsto \frac{\partial^{2}}{\partial x^{2}} \mu(x, \theta),(x, \theta) \mapsto \frac{\partial}{\partial x} \mu(x, \theta),(x, \theta) \mapsto \frac{\partial}{\partial_{\theta_{i}}} \frac{\partial}{\partial_{x}} \frac{\partial}{\partial_{\theta_{j}}} \mu(x, \theta)$ are continuous on $E \times C l(\Theta)$, for all $i, j=1, \ldots, d$. Function $x \mapsto \nu(x)$ is two-times continuously differentiable on $E$, and $\nu(x)>0$ for all $x \in E$.

(A3) For all $m \leq d+3$, there exist partial derivatives $D_{\theta}^{m} \mu(x, \theta)$ and $\frac{\partial}{\partial x} D_{\theta}^{m} \mu(x, \theta)$ on $E \times C l(\Theta)$. Furthermore, functions $(x, \theta) \mapsto D_{\theta}^{m} \mu(x, \theta)$, $(x, \theta) \mapsto \frac{\partial}{\partial x} D_{\theta}^{m} \mu(x, \theta), m \leq d+3$, are continuous on $E \times C l(\Theta)$.

Let

$$
L_{T}(\theta)=\int_{0}^{T} \frac{\mu\left(X_{s}, \theta\right)}{\sigma_{0}^{2} \nu^{2}\left(X_{s}\right)} d X_{s}-\frac{1}{2} \int_{0}^{T} \frac{\mu^{2}\left(X_{s}, \theta\right)}{\sigma_{0}^{2} \nu^{2}\left(X_{s}\right)} d s
$$

be a continuous-time log-likelihood function, shortly LLF (see [5]).

(A4) For all $\omega \in \Omega$, LLF $\theta \mapsto L_{T}(\theta) \equiv L_{T}(\omega, \theta)$ has a unique point of global maximum $\hat{\theta}(\omega) \in \Theta$, and $D^{2} L_{T}(\hat{\theta})<0$ which means that Hessian $D^{2} L_{T}(\hat{\theta})$ is a negatively definite matrix.

For example, the general growth diffusion process (see [8]) satisfies assumptions (A1-3) obviously, and (A4) on an event which probability tends to unity. Generally, these assumptions (and in the same sense) satisfies any ergodic diffusion such that (H1B-5B) from [10] hold. More precisely, these diffusions have a property that a.s. there exists a time $\tau>0$ such that for all times $T \geq \tau$, (A4) holds. Diffusions that satisfy (A4) with certainty are those that satisfy (A1-3) and have drift functions that are linear combinations of linearly independent functions defined on state space with coefficients equal to drift parameters such that the values of (vector) parameters are in $\Theta=\mathbb{R}^{d}$ (see [3]). For such diffusions we will say briefly that are linear in drift parameters. To prove that the main results of this paper hold for diffusions linear in drift parameters we do not need the assumption of relatively compactness of drift parameter space (see Remark 5.5).

For each $\theta \in \Theta$ let $\Sigma(\theta)$ be $d \times d$ random matrix which $j k$ component is defined by

$$
\Sigma(\theta)^{j k}=\frac{1}{2} \int_{0}^{T} \nu^{4}\left(X_{s}\right) \frac{\partial}{\partial x} \frac{\frac{\partial}{\partial \theta_{j}} \mu\left(X_{s}, \theta\right)}{\nu^{2}\left(X_{s}\right)} \frac{\partial}{\partial x} \frac{\frac{\partial}{\partial \theta_{k}} \mu\left(X_{s}, \theta\right)}{\nu^{2}\left(X_{s}\right)} d s .
$$

Under conditions (A1)-(A4) in [10] is proved that there exists a sequence $\left(\hat{\theta}_{n}, n \in \mathbb{N}\right) \subseteq \Theta$ of $\mathcal{F}_{T}$-measurable random vectors such that the following 
hold:

$$
\begin{aligned}
& \lim _{n} \mathbb{P}\left\{D L_{n}\left(\hat{\theta}_{n}\right)=0\right\}=1, \\
& \hat{\theta}_{n} \stackrel{\mathbb{P}}{\rightarrow} \hat{\theta}, n \rightarrow \infty,
\end{aligned}
$$

if $\left(\tilde{\theta}_{n}, n \in \mathbb{N}\right)$ is any other sequence of random vectors which satisfies (4.2) and (4.3), then $\lim _{n} \mathbb{P}\left\{\tilde{\theta}_{n}=\hat{\theta}_{n}\right\}=1$, $\left(\frac{1}{\sqrt{\Delta_{n}}}\left\|\hat{\theta}_{n}-\hat{\theta}\right\|, n \in \mathbb{N}\right)$ is bounded in probability.

(4.2)-(4.5) are consequences of

$$
\sup _{\theta \in \Theta}\left\|D^{r} L_{n}(\theta)-D^{r} L_{T}(\theta)\right\|=O_{\mathbb{P}}\left(\sqrt{\Delta_{n}}\right), \quad n \in \mathbb{N} r=1,2,
$$

and so called the general theorem on approximate maximum likelihood estimation (see [9]). In the proof of this theorem $\hat{\theta}_{n}$ is constructed to be a point of global maximum of $L_{n}$ in $\Theta$ on an event with probability tending to unity, and hence we can take $\bar{\theta}_{n}=\hat{\theta}_{n}$ on this event. It is sufficient to assume that $\left(\bar{\theta}_{n}, n \in \mathbb{N}\right)$ satisfies (4.2)-(4.5), and all of our results are stated for that kind of sequence.

TheOrem 4.1. Assume that (A1)-(A4) hold. Assume that $\left(\bar{\theta}_{n}, n \in \mathbb{N}\right)$ satisfies (4.2)-(4.5). Then

$$
\frac{1}{\sqrt{\Delta_{n}}}\left(\bar{\theta}_{n}-\hat{\theta}\right) \stackrel{s t}{\Rightarrow} M N\left(0,\left(D^{2} L_{T}(\hat{\theta})\right)^{-1} \Sigma(\hat{\theta})\left(D^{2} L_{T}(\hat{\theta})\right)^{-1}\right) .
$$

For real symmetric $d \times d$ matrix $C$ let us denote by $\lambda_{k}(C)$ its $k$-th in order eigenvalue in the ordered sequence of eigenvalues $\lambda_{1}(C) \leq \lambda_{2}(C) \leq$ $\cdots \leq \lambda_{d}(C)$. Let us additionally assume:

(A5) For all $\omega \in \Omega$, matrix $\Sigma(\omega, \hat{\theta}(\omega))$ is regular. There exists a constant $\lambda_{\Sigma}>0$ such that $\lambda_{1}\left(\Sigma(\omega, \hat{\theta}(\omega)) \geq \lambda_{\Sigma}\right.$, for all $\omega \in \Omega$.

For $n \in \mathbb{N}$ let us define random matrix $\Sigma_{n}(\theta)$ by defining its $j k$ - component in the following way:

$$
\Sigma_{n}(\theta)^{j k}=\sum_{i=1}^{n} \frac{1}{2} \nu^{4}\left(X_{t_{i-1}}\right) \partial_{x}\left(\frac{\partial \theta_{j} \mu\left(X_{t_{i-1}}, \theta\right)}{\nu^{2}\left(X_{t_{i-1}}\right)}\right) \partial_{x}\left(\frac{\partial \theta_{k} \mu\left(X_{t_{i-1}}, \theta\right)}{\nu^{2}\left(X_{t_{i-1}}\right)}\right) \Delta_{n},
$$

where $\partial \theta_{j}:=\frac{\partial}{\partial \theta_{j}}, j=1, \ldots, d$. It is obvious, from the definition, that $\Sigma_{n}(\theta)^{j k}$ can be considered as a discretization of $\Sigma(\theta)^{j k}$.

Corollary 4.2. Assume that (A1)-(A5) hold. Assume that $\left(\bar{\theta}_{n}, n \in \mathbb{N}\right)$ satisfies (4.2)-(4.5). Then

$$
\left(\sqrt{\Sigma_{n}\left(\bar{\theta}_{n}\right)}\right)^{-1} D^{2} L_{n}\left(\bar{\theta}_{n}\right) \frac{1}{\sqrt{\Delta_{n}}}\left(\bar{\theta}_{n}-\hat{\theta}\right) 1_{\left\{\Sigma_{n}\left(\bar{\theta}_{n}\right) \text { is regular matrix }\right\}} \stackrel{s t}{\Rightarrow} N(0, I) .
$$


REMARK 4.3. In the case when the subdivision of the segment $[0, T]$ is not equidistant, all of the results will still hold under the assumptions that $\lim _{n} \Delta_{n}=0$ and $\lim _{n \rightarrow \infty} \max _{i=1, \ldots, n}\left|\frac{h_{i}-\Delta_{n}}{\Delta_{n}}\right|=0$, where $h_{i}:=t_{i}-t_{i-1}, i=$ $1, \ldots, n, \Delta_{n}:=\max _{1 \leq i \leq n} h_{i}$, and by replacing $\Sigma_{n}(\theta)$ with $\tilde{\Sigma}_{n}(\theta)$ such that

$$
\tilde{\Sigma}_{n}(\theta)^{j k}=\sum_{i=1}^{n} \frac{1}{2} \nu^{4}\left(X_{t_{i-1}}\right) \partial_{x}\left(\frac{\partial \theta_{j} \mu\left(X_{t_{i-1}}, \theta\right)}{\nu^{2}\left(X_{t_{i-1}}\right)}\right) \partial_{x}\left(\frac{\partial \theta_{k} \mu\left(X_{t_{i-1}}, \theta\right)}{\nu^{2}\left(X_{t_{i-1}}\right)}\right) h_{i} \text {. }
$$

\section{ProOFs}

We will prove Theorem 4.1 in number of steps. Some of the lemmas will be proved in Appendix.

Let $\hat{\theta}$ be a continuous-time MLE, and $\bar{\theta}_{n}$ be an AMLE which satisfies (4.2)-(4.5). Using mean value theorem for the function $D L_{n}$ which is, due to (A2), continuously differentiable on $\Theta$, for all $\omega \in \Omega$, we get

$$
D L_{n}(\theta)=D L_{n}(\hat{\theta})+A_{\hat{\theta}, \theta}(\theta-\hat{\theta}),
$$

for all $\theta \in \Theta$, where $A_{\hat{\theta}, \theta}:=\int_{0}^{1} D^{2} L_{n}(\hat{\theta}+v(\theta-\hat{\theta})) d v$, and integration is by components. If we put $\theta=\bar{\theta}_{n}$, then on the event $\left\{D L_{n}\left(\bar{\theta}_{n}\right)=0\right\}$ we have

$$
0=D L_{n}\left(\bar{\theta}_{n}\right)=D L_{n}(\hat{\theta})+A_{\hat{\theta}, \bar{\theta}_{n}}\left(\bar{\theta}_{n}-\hat{\theta}\right),
$$

where $A_{\hat{\theta}, \bar{\theta}_{n}}:=\int_{0}^{1} D^{2} L_{n}\left(\hat{\theta}+v\left(\bar{\theta}_{n}-\hat{\theta}\right)\right) d v$. Since $D L_{T}(\hat{\theta})=0$, we can write

$$
A_{\hat{\theta}, \bar{\theta}_{n}}\left(\bar{\theta}_{n}-\hat{\theta}\right)=-D L_{n}(\hat{\theta})=D L_{T}(\hat{\theta})-D L_{n}(\hat{\theta})
$$

which implies

$$
A_{\hat{\theta}, \bar{\theta}_{n}} \frac{1}{\sqrt{\Delta_{n}}}\left(\bar{\theta}_{n}-\hat{\theta}\right)=\frac{1}{\sqrt{\Delta_{n}}}\left(D L_{T}(\hat{\theta})-D L_{n}(\hat{\theta})\right)
$$

on the event $\left\{D L_{n}\left(\bar{\theta}_{n}\right)=0\right\}$.

First, in Theorem 5.3 we will prove that the right hand side of equation (5.1) converges stably in law, for fixed parameter $\theta \in \Theta$, and then in Theorem 5.4 we will prove the same result but for MLE $\hat{\theta}$. After that we will prove Theorem 4.1 .

Let $\theta \in \Theta$ be fixed, arbitrary parameter value. Let us denote $X_{i}:=$ $X_{t_{i}}, i=0, \ldots, n$, and $\partial \theta_{j}:=\frac{\partial}{\partial \theta_{j}}, j=1, \ldots, d$. Define functions

$$
g_{j}(x)=g_{j}(x, \theta):=\frac{\partial \theta_{j} \mu(x, \theta)}{\nu^{2}(x)}, j=1, \ldots, d,
$$

and denote by $g_{j}^{\prime}$ and $g_{j}^{\prime \prime}$ its first and second derivative with respect to $x$. Define functions

$$
f_{j}(x)=f_{j}(x, \theta):=\frac{\mu(x, \theta) \partial \theta_{j} \mu(x, \theta)}{\nu^{2}(x)}, j=1, \ldots, d,
$$

and denote by $f_{j}^{\prime}$ and $f_{j}^{\prime \prime}$ its first and second derivative with respect to $x$. 
(A6) Functions $\mu, \nu, \frac{1}{\nu}, g_{j}, g_{j}^{\prime}, g_{j}^{\prime \prime}, f_{j}, f_{j}^{\prime}, f_{j}^{\prime \prime}, \frac{\partial}{\partial x} \mu, \frac{\partial^{2}}{\partial x^{2}} \mu, \nu^{\prime}, \nu^{\prime \prime}$ are bounded.

REMARK 5.1. The assumption (A6) is only here for technical reasons. First, we will prove our results under assumption (A6), and then we will prove our results without that assumption.

Lemma 5.2. Assume that (A1)-(A4), (A6) hold. Then, for arbitrary fixed $\theta \in \Theta$ we have

$$
\begin{aligned}
& \frac{1}{\sqrt{\Delta_{n}}}\left(D L_{T}(\theta)-D L_{n}(\theta)\right)=Z_{n}(\theta) \\
& +\frac{1}{\sqrt{\Delta_{n}}}\left[\begin{array}{c}
\sum_{i=1}^{n} \int_{t_{i-1}}^{t_{i}} \nu\left(X_{s}\right) \int_{t_{i-1}}^{s} g_{1}^{\prime}\left(X_{u}\right) \nu\left(X_{u}\right) d W_{u} d W_{s} \\
\vdots \\
\sum_{i=1}^{n} \int_{t_{i-1}}^{t_{i}} \nu\left(X_{s}\right) \int_{t_{i-1}}^{s} g_{d}^{\prime}\left(X_{u}\right) \nu\left(X_{u}\right) d W_{u} d W_{s}
\end{array}\right],
\end{aligned}
$$

where $Z_{n}(\theta) \stackrel{\mathbb{P}}{\rightarrow} 0, n \rightarrow+\infty$.

Proof. For details see the Appendix.

TheOREM 5.3. Assume that (A1)-(A4) hold. Then, for arbitrary fixed parameter $\theta \in \Theta$ we have

$$
\frac{1}{\sqrt{\Delta_{n}}}\left(D L_{T}(\theta)-D L_{n}(\theta)\right) \stackrel{s t}{\Rightarrow} Y(\theta)
$$

where $Y(\theta) \sim M N(0, \Sigma(\theta))$.

Proof. First, let us assume that (A6) holds.

Let us denote

$$
R_{t}^{j}(\theta):=g_{j}^{\prime}\left(X_{t}\right) \nu\left(X_{t}\right)=g_{j}^{\prime}\left(X_{t}, \theta\right) \nu\left(X_{t}\right)
$$

hence

$$
\Sigma(\theta)^{j k}=\frac{1}{2} \int_{0}^{T} \nu^{2}\left(X_{s}\right) R_{s}^{j}(\theta) R_{s}^{k}(\theta) d s, \quad j, k=1, \ldots, d .
$$

Let us define continuous adapted process $C=\left(C_{t}^{j k}\right)_{0 \leq t \leq T}$ by

$$
C_{t}^{j k}=\frac{1}{2} \int_{0}^{t} \nu^{2}\left(X_{s}\right) R_{s}^{j}(\theta) R_{s}^{k}(\theta) d s, \quad j, k=1, \ldots, d, t \in[0, T],
$$

and define $\mathcal{F}_{t_{i}}$-measurable random vectors $\chi_{i}^{n}, i=1, \ldots, n$ by

$$
\chi_{i}^{n}=\left[\begin{array}{c}
\chi_{i}^{n, 1} \\
\vdots \\
\chi_{i}^{n, d}
\end{array}\right]=\frac{1}{\sqrt{\Delta_{n}}}\left[\begin{array}{c}
\int_{t_{i-1}}^{t_{i}} \nu\left(X_{s}\right) \int_{t_{i-1}}^{s} R_{u}^{1}(\theta) d W_{u} d W_{s} \\
\vdots \\
\int_{t_{i-1}}^{t_{i}} \nu\left(X_{s}\right) \int_{t_{i-1}}^{s} R_{u}^{d}(\theta) d W_{u} d W_{s}
\end{array}\right] .
$$

We will prove that for $C$ and $\chi_{i}^{n}$ Theorem 2.1 holds. Random vectors $\chi_{i}^{n}$ are square integrable due to (A6). Because of its definition, $C_{t}$ is symmetric 
positive semidefinite random matrix, for all $t \in[0, T]$. Using notation $\mathcal{F}_{n, i}:=$ $\mathcal{F}_{t_{i}}, i=1, \ldots, n$, we have

$$
\mathbb{E}\left[\chi_{i}^{n, j} \mid \mathcal{F}_{n, i-1}\right]=0, \quad \forall j=1, \ldots, d, \forall i=1, \ldots, n,
$$

hence $(2.1)$ is trivially satisfied.

Let $\epsilon>0$. Then, there exists constant $k_{9}>0$, such that

$$
\left\|\sum_{i=1}^{n} \mathbb{E}\left[\left\|\chi_{i}^{n}\right\|^{2} 1_{\left\{\left\|\chi_{i}^{n}\right\|>\epsilon\right\}} \mid \mathcal{F}_{n, i-1}\right]\right\|_{1} \leq \frac{d}{\epsilon^{2}} \sum_{i=1}^{n} \mathbb{E}\left[\sum_{j=1}^{d}\left(\chi_{i}^{n, j}\right)^{4}\right] \leq k_{9} \Delta_{n} T,
$$

hence (2.4) is satisfied. The last inequality and existence of $k_{9}$ follows in a way similar to the proof of the existence of constant $k_{2}$ (see the proof of Lemma 5.2 in Appendix).

Let $N=\left(N_{t}\right)_{0 \leq t \leq T}$ be any bounded $\mathcal{F}_{t}$-martingal orthogonal to $W$. Since $\left(\mathcal{F}_{t}\right)_{0 \leq t \leq T}$ is generated by Brownian motion $W$, it follows from martingale representation theorem (see [13, Theorem III.4.33]) that any $\mathcal{F}_{t^{-}}$martingale can be represented as the sum of constant term and a stochastic integral with respect to $W$. Since $N_{t}$ is bounded $\mathcal{F}_{t}$-martingale orthogonal to $W$, it follows that $N_{t}$ is equal to constant, so $(2.5)$ is satisfied.

Let us recall that $\theta_{0}$ is the true value of (vector) drift parameter. For $j=1, \ldots, d$, we have

$$
\begin{aligned}
& \sum_{i=1}^{A_{n}^{t}} \mathbb{E} {\left[\chi_{i}^{n, j}\left(W_{t_{i}}-W_{t_{i-1}}\right) \mid \mathcal{F}_{n, i-1}\right] } \\
&= \frac{1}{\sqrt{\Delta_{n}}} \sum_{i=1}^{A_{n}^{t}} \mathbb{E}\left[\int_{t_{i-1}}^{t_{i}} \nu\left(X_{s}\right) \int_{t_{i-1}}^{s} R_{u}^{j}(\theta) d W_{u} d s \mid \mathcal{F}_{n, i-1}\right] \\
&= \frac{1}{\sqrt{\Delta_{n}}} \sum_{i=1}^{A_{n}^{t}} \mathbb{E}\left[\int_{t_{i-1}}^{t_{i}}\left(\int_{t_{i-1}}^{s}\left(\nu^{\prime}\left(X_{u}\right) \mu\left(X_{u}, \theta_{0}\right)+\frac{1}{2} \nu^{\prime \prime}\left(X_{u}\right) \sigma_{0}^{2} \nu^{2}\left(X_{u}\right)\right) d u\right) .\right. \\
&\left.(5.3) \quad \cdot\left(\int_{t_{i-1}}^{s} R_{u}^{j}(\theta) d W_{u} d s\right) \mid \mathcal{F}_{n, i-1}\right] \\
& \quad+\frac{1}{\sqrt{\Delta_{n}}} \sum_{i=1}^{A_{n}^{t}} \mathbb{E}\left[\int_{t_{i-1}}^{t_{i}}\left(\int_{t_{i-1}}^{s} \nu^{\prime}\left(X_{u}\right) \sigma_{0} \nu\left(X_{u}\right) d W_{u}\right) \cdot\left(\int_{t_{i-1}}^{s} R_{u}^{j}(\theta) d W_{u} d s\right) \mid \mathcal{F}_{n, i-1}\right] .
\end{aligned}
$$

If $\nu \equiv c>0$ is a constant function, then (2.3) is trivially satisfied, because

$$
\begin{aligned}
\frac{1}{\sqrt{\Delta_{n}}} & \sum_{i=1}^{A_{n}^{t}} \mathbb{E}\left[\int_{t_{i-1}}^{t_{i}} \nu\left(X_{s}\right) \int_{t_{i-1}}^{s} R_{u}^{j}(\theta) d W_{u} d s \mid \mathcal{F}_{n, i-1}\right] \\
& =\frac{c}{\sqrt{\Delta_{n}}} \sum_{i=1}^{A_{n}^{t}} \mathbb{E}\left[\int_{t_{i-1}}^{t_{i}} \int_{t_{i-1}}^{s} R_{u}^{j}(\theta) d W_{u} d s \mid \mathcal{F}_{n, i-1}\right]=0 .
\end{aligned}
$$


So, let us assume that $\nu$ is not a constant function. There exist constants $k_{10}, k_{11}$ such that

$$
\begin{aligned}
& \| \frac{1}{\sqrt{\Delta_{n}}} \sum_{i=1}^{A_{n}^{t}} \mathbb{E}\left[\int_{t_{i-1}}^{t_{i}}\left(\int_{t_{i-1}}^{s}\left(\nu^{\prime}\left(X_{u}\right) \mu\left(X_{u}, \theta_{0}\right)+\frac{1}{2} \nu^{\prime \prime}\left(X_{u}\right) \sigma_{0}^{2} \nu^{2}\left(X_{u}\right)\right) d u\right) .\right. \\
& \left.\cdot\left(\int_{t_{i-1}}^{s} R_{u}^{j}(\theta) d W_{u}\right) d s \mid \mathcal{F}_{n, i-1}\right] \|_{1} \leq k_{10} \sqrt{\Delta_{n}} T
\end{aligned}
$$

and

$$
\begin{aligned}
& \left|\frac{1}{\sqrt{\Delta_{n}}} \sum_{i=1}^{A_{n}^{t}} \mathbb{E}\left[\int_{t_{i-1}}^{t_{i}}\left(\int_{t_{i-1}}^{s} \nu^{\prime}\left(X_{u}\right) \sigma_{0} \nu\left(X_{u}\right) d W_{u}\right) \cdot\left(\int_{t_{i-1}}^{s} R_{u}^{j}(\theta) d W_{u}\right) d s \mid \mathcal{F}_{n, i-1}\right]\right| \\
& \quad=\left|\frac{1}{\sqrt{\Delta_{n}}} \sum_{i=1}^{A_{n}^{t}} \mathbb{E}\left[\int_{t_{i-1}}^{t_{i}} \int_{t_{i-1}}^{s} \nu^{\prime}\left(X_{u}\right) \sigma_{0} \nu\left(X_{u}\right) R_{u}^{j}(\theta) d u d s \mid \mathcal{F}_{n, i-1}\right]\right| \\
& \quad \leq k_{11} \sqrt{\Delta_{n}} T
\end{aligned}
$$

so (5.3) converges in $L^{1}$-norm to zero and (5.4) converges almost surely to zero, hence (2.3) is satisfied. For example, for proving the existence of constant $k_{10}$ let us define $M:=\sup _{0 \leq s \leq T}\left|\int_{0}^{s} R_{u}^{j}(\theta) d W_{u}\right|$. We have

$$
\begin{aligned}
& \mathbb{E}\left[\mid \frac{1}{\sqrt{\Delta_{n}}} \sum_{i=1}^{A_{n}^{t}} \mathbb{E}\left[\int_{t_{i-1}}^{t_{i}}\left(\int_{t_{i-1}}^{s}\left(\nu^{\prime}\left(X_{u}\right) \mu\left(X_{u}, \theta_{0}\right)+\frac{1}{2} \nu^{\prime \prime}\left(X_{u}\right) \sigma_{0}^{2} \nu^{2}\left(X_{u}\right)\right) d u\right) \cdot\right.\right. \\
& \left.\left.\cdot\left(\int_{t_{i-1}}^{s} R_{u}^{j}(\theta) d W_{u}\right) d s \mid \mathcal{F}_{n, i-1}\right] \mid\right] \\
& \leq \frac{1}{\sqrt{\Delta_{n}}} \sum_{i=1}^{A_{n}^{t}} \mathbb{E}\left[\mid \int_{t_{i-1}}^{t_{i}}\left(\int_{t_{i-1}}^{s}\left(\nu^{\prime}\left(X_{u}\right) \mu\left(X_{u}, \theta_{0}\right)+\frac{1}{2} \nu^{\prime \prime}\left(X_{u}\right) \sigma_{0}^{2} \nu^{2}\left(X_{u}\right)\right) d u\right)\right. \\
& \left.\quad \cdot\left(\int_{t_{i-1}}^{s} R_{u}^{j}(\theta) d W_{u}\right) d s \mid\right] \\
& \leq \frac{\text { const }_{1}}{\sqrt{\Delta_{n}}} \sum_{i=1}^{A_{n}^{t}} \int_{t_{i-1}}^{t_{i}}\left(s-t_{i-1}\right) \mathbb{E}[M] d s \leq \text { const }_{2} \sqrt{\Delta_{n}} T \mathbb{E}[M]
\end{aligned}
$$

From Doob's inequality we conclude that $\mathbb{E}[M]<+\infty$, and we define $k_{10}:=$ const $_{2} \mathbb{E}[M]$. 
It is only left to prove that (2.2) is satisfied. Let $1 \leq j \leq k \leq d$. Then

$$
\begin{aligned}
\sum_{i=1}^{A_{n}^{t}}\left(\mathbb{E}\left[\chi_{i}^{n, j} \chi_{i}^{n, k} \mid \mathcal{F}_{n, i-1}\right]-\mathbb{E}\left[\chi_{i}^{n, j} \mid \mathcal{F}_{n, i-1}\right] \mathbb{E}\left[\chi_{i}^{n, k} \mid \mathcal{F}_{n, i-1}\right]\right) \\
=\frac{1}{\Delta_{n}} \sum_{i=1}^{A_{n}^{t}} \mathbb{E}\left[\int_{t_{i-1}}^{t_{i}} \nu^{2}\left(X_{s}\right) \int_{t_{i-1}}^{s} J_{u}^{(i, j)} R_{u}^{k}(\theta) d W_{u} d s \mid \mathcal{F}_{n, i-1}\right] \\
\quad+\frac{1}{\Delta_{n}} \sum_{i=1}^{A_{n}^{t}} \mathbb{E}\left[\int_{t_{i-1}}^{t_{i}} \nu^{2}\left(X_{s}\right) \int_{t_{i-1}}^{s} J_{u}^{(i, k)} R_{u}^{j}(\theta) d W_{u} d s \mid \mathcal{F}_{n, i-1}\right] \\
\quad+\frac{1}{\Delta_{n}} \sum_{i=1}^{A_{n}^{t}} \mathbb{E}\left[\int_{t_{i-1}}^{t_{i}} \nu^{2}\left(X_{s}\right) \int_{t_{i-1}}^{s} R_{u}^{j}(\theta) R_{u}^{k}(\theta) d u d s \mid \mathcal{F}_{n, i-1}\right]
\end{aligned}
$$

where $J_{s}^{(i, j)}:=\int_{t_{i-1}}^{s} R_{u}^{j}(\theta) d W_{u}$. If $\nu$ is constant function, then (5.5) and (5.6) are equal to zero, and the proof that (5.7) converges in probability to $C_{t}^{j k}, \forall t \in[0, T]$ would stay the same as in the rest of the proof. So, let us assume that $\nu$ is not a constant function. It can be shown that there exist constants $k_{12}, k_{13}$, such that

$$
\begin{aligned}
& \left\|\frac{1}{\Delta_{n}} \sum_{i=1}^{A_{n}^{t}} \mathbb{E}\left[\int_{t_{i-1}}^{t_{i}} \nu^{2}\left(X_{s}\right) \int_{t_{i-1}}^{s} J_{u}^{(i, j)} R_{u}^{k}(\theta) d W_{u} d s \mid \mathcal{F}_{n, i-1}\right]\right\|_{2} \leq k_{12} \sqrt{\Delta_{n}}(T+\sqrt{T}), \\
& \left\|\frac{1}{\Delta_{n}} \sum_{i=1}^{A_{n}^{t}} \mathbb{E}\left[\int_{t_{i-1}}^{t_{i}} \nu^{2}\left(X_{s}\right) \int_{t_{i-1}}^{s} J_{u}^{(i, k)} R_{u}^{j}(\theta) d W_{u} d s \mid \mathcal{F}_{n, i-1}\right]\right\|_{2} \leq k_{13} \sqrt{\Delta_{n}}(T+\sqrt{T}),
\end{aligned}
$$

so (5.5) and (5.6) converge in probability to zero. It is left to prove that (5.7) converges in probability to $C_{t}^{j k}$. There exists constant $k_{14}$ such that

$$
\begin{aligned}
\frac{1}{\Delta_{n}} \sum_{i=1}^{A_{n}^{t}} \mathbb{E}\left[\int_{t_{i-1}}^{t_{i}} \nu^{2}\left(X_{s}\right) \int_{t_{i-1}}^{s} R_{u}^{j}(\theta) R_{u}^{k}(\theta) d u d s \mid \mathcal{F}_{n, i-1}\right]= \\
=\frac{1}{\Delta_{n}} \sum_{i=1}^{A_{n}^{t}}\left(\mathbb{E}\left[\int_{t_{i-1}}^{t_{i}} \nu^{2}\left(X_{s}\right) \int_{t_{i-1}}^{s} R_{u}^{j}(\theta) R_{u}^{k}(\theta) d u d s \mid \mathcal{F}_{n, i-1}\right]\right. \\
\left.\quad-\int_{t_{i-1}}^{t_{i}} \nu^{2}\left(X_{s}\right) \int_{t_{i-1}}^{s} R_{u}^{j}(\theta) R_{u}^{k}(\theta) d u d s\right) \\
\quad+\frac{1}{\Delta_{n}} \sum_{i=1}^{A_{n}^{t}} \int_{t_{i-1}}^{t_{i}} \nu^{2}\left(X_{s}\right) \int_{t_{i-1}}^{s} R_{u}^{j}(\theta) R_{u}^{k}(\theta) d u d s
\end{aligned}
$$


and

$$
\begin{aligned}
& \| \frac{1}{\Delta_{n}} \sum_{i=1}^{A_{n}^{t}}\left(\mathbb{E}\left[\int_{t_{i-1}}^{t_{i}} \nu^{2}\left(X_{s}\right) \int_{t_{i-1}}^{s} R_{u}^{j}(\theta) R_{u}^{k}(\theta) d u d s \mid \mathcal{F}_{n, i-1}\right]\right. \\
& \left.\quad-\int_{t_{i-1}}^{t_{i}} \nu^{2}\left(X_{s}\right) \int_{t_{i-1}}^{s} R_{u}^{j}(\theta) R_{u}^{k}(\theta) d u d s\right) \|_{2} \leq k_{14} \sqrt{\Delta_{n}} \sqrt{T}
\end{aligned}
$$

hence (5.8) converges in probability to zero.

We will show that (5.9) converges in probability to $C_{t}^{j k}$. Let

$$
l(u, s)(\omega):=\left(\nu^{2}\left(X_{s}\right) R_{u}^{j}(\theta) R_{u}^{k}(\theta)\right)(\omega)
$$

be a function defined on $[0, T] \times[0, T] \times \Omega$. For fixed $\omega \in \Omega$ function $l(u, s)(\omega)$ is bounded, continuous function on $[0, T] \times[0, T]$, which means that there exist $u_{i}^{*}(\omega), s_{i}^{*}(\omega) \in\left[t_{i-1}, t_{i}\right]$ such that $u_{i}^{*}(\omega) \leq s_{i}^{*}(\omega)$ and

$$
\int_{t_{i-1}}^{t_{i}} \int_{t_{i-1}}^{s} l(u, s) d u d s=\frac{\Delta_{n}^{2}}{2} l\left(u_{i}^{*}, s_{i}^{*}\right), \quad i \in\{1, \ldots, n\} .
$$

Hence,

$$
\begin{aligned}
& \frac{1}{\Delta_{n}} \sum_{i=1}^{A_{n}^{t}} \int_{t_{i-1}}^{t_{i}} \nu^{2}\left(X_{s}\right) \int_{t_{i-1}}^{s} R_{u}^{j}(\theta) R_{u}^{k}(\theta) d u d s-C_{t}^{j k} \\
& =\sum_{i=1}^{A_{n}^{t}} \frac{\Delta_{n}}{2} l\left(u_{i}^{*}, u_{i}^{*}\right)-\frac{1}{2} \int_{0}^{t} \nu^{2}\left(X_{s}\right) R_{s}^{j}(\theta) R_{s}^{k}(\theta) d s \\
& \quad+\sum_{i=1}^{A_{n}^{t}} \frac{\Delta_{n}}{2}\left(l\left(u_{i}^{*}, s_{i}^{*}\right)-l\left(u_{i}^{*}, u_{i}^{*}\right)\right) .
\end{aligned}
$$

Continuity of integrand function assures that there exist some $t^{*}(\omega) \in\left[t_{A_{n}^{t}}, t\right]$, such that $\frac{1}{2} \int_{t_{A_{n}^{t}}^{t}}^{t} \nu^{2}\left(X_{s}\right) R_{s}^{j}(\theta) R_{s}^{k}(\theta) d s=\frac{1}{2}\left(t-t_{A_{n}^{t}}\right) \nu^{2}\left(X_{t^{*}}\right) R_{t^{*}}^{j}(\theta) R_{t^{*}}^{k}(\theta)$, therefore for (5.10) we have

$$
\begin{aligned}
\sum_{i=1}^{A_{n}^{t}} & \frac{\Delta_{n}}{2} l\left(u_{i}^{*}, u_{i}^{*}\right)-\frac{1}{2} \int_{0}^{t} \nu^{2}\left(X_{s}\right) R_{s}^{j}(\theta) R_{s}^{k}(\theta) d s= \\
= & \sum_{i=1}^{A_{n}^{t}} \frac{\Delta_{n}}{2} \nu^{2}\left(X_{u_{i}^{*}}\right) R_{u_{i}^{*}}^{j}(\theta) R_{u_{i}^{*}}^{k}(\theta)+\frac{1}{2}\left(t-t_{A_{n}^{t}}\right) \nu^{2}\left(X_{t^{*}}\right) R_{t^{*}}^{j}(\theta) R_{t^{*}}^{k}(\theta) \\
& -\frac{1}{2} \int_{0}^{t} \nu^{2}\left(X_{s}\right) R_{s}^{j}(\theta) R_{s}^{k}(\theta) d s-\frac{1}{2} \int_{t_{A_{n}^{t}}}^{t} \nu^{2}\left(X_{s}\right) R_{s}^{j}(\theta) R_{s}^{k}(\theta) d s \stackrel{\text { a.s. }}{\rightarrow} 0
\end{aligned}
$$


hence (5.10) converges in probability to zero. For (5.11) there exists constant $k_{15}$ such that we have

$$
\begin{aligned}
& \left|\sum_{i=1}^{A_{n}^{t}} \frac{\Delta_{n}}{2}\left(l\left(u_{i}^{*}, s_{i}^{*}\right)-l\left(u_{i}^{*}, u_{i}^{*}\right)\right)\right|=\left|\sum_{i=1}^{A_{n}^{t}} \frac{\Delta_{n}}{2}\left(\nu^{2}\left(X_{s_{i}^{*}}\right)-\nu^{2}\left(X_{u_{i}^{*}}\right)\right) R_{u_{i}^{*}}^{j}(\theta) R_{u_{i}^{*}}^{k}(\theta)\right| \\
& \quad \leq k_{15} \sum_{i=1}^{A_{n}^{t}} \frac{\Delta_{n}}{2}\left|\nu^{2}\left(X_{s_{i}^{*}}\right)-\nu^{2}\left(X_{u_{i}^{*}}\right)\right| .
\end{aligned}
$$

Let $\epsilon>0$. For fixed $\omega \in \Omega$ the function $t \mapsto \nu^{2}\left(X_{t}(\omega)\right)$ is continuous on $[0, T]$, so it is uniformly continuous. It means that

$$
(\exists \delta>0)(\forall s, t \in[0, T])(|s-t|<\delta) \Rightarrow\left|\nu^{2}\left(X_{s}(\omega)\right)-\nu^{2}\left(X_{t}(\omega)\right)\right|<\epsilon .
$$

We omitted writing $\omega$, because it is fixed. $\lim _{n \rightarrow \infty} \Delta_{n}=0$, so there exists $n_{0} \in \mathbb{N}$ such that for all $n \geq n_{0}$ holds $\Delta_{n}<\delta$. Then, for all $n \geq n_{0}$ we have

$$
\sum_{i=1}^{A_{n}^{t}} \frac{\Delta_{n}}{2}\left|\nu^{2}\left(X_{s_{i}^{*}}\right)-\nu^{2}\left(X_{u_{i}^{*}}\right)\right| \leq \epsilon \frac{T}{2},
$$

which means that (5.11) converges almost surely to zero 0 , so it converges in probability to zero.

Therefore, conditions of Theorem 2.1 are satisfied provided that (A6) holds. If we denote by $\pi_{T}$ projection function $\pi_{T}: D\left([0, T], \mathbb{R}^{d}\right) \rightarrow \mathbb{R}^{d}$, defined by $\pi_{T}\left(\left(X_{s}, s \in[0, T]\right)\right):=X_{T}$, then by $[2$, Theorem 12.5$]$ projection $\pi_{T}$ is continuous function. Notice that

$$
\frac{1}{\sqrt{\Delta_{n}}}\left(D L_{T}(\theta)-D L_{n}(\theta)\right)=\pi_{T}\left(\left(\sum_{i=1}^{A_{n}^{t}} \chi_{i}^{n}, t \in[0, T]\right)\right)+Z_{n}(\theta),
$$

hence by definition and properties of stable convergence in law, our theorem holds under assumption (A6).

In general case (i.e. without assumption (A6)), let $\left(E_{M}, M \in \mathbb{N}\right)$ be a sequence of open and relatively compact subsets of $E$ such that $x_{0} \in E_{1}$, $C l\left(E_{M}\right) \subseteq E_{M+1}, \forall M \in \mathbb{N}$ and $\cup_{M=1}^{\infty} E_{M}=E$. For that sequence and the solution $\left(X_{t}, t \geq 0\right)$ of our $\operatorname{SDE}(1.1)$ define for $M \in \mathbb{N}$

$$
T_{M}:=\inf \left\{t \geq 0: X_{t} \in E_{M}^{c}\right\},
$$

where $\inf \emptyset=+\infty$. Since $X$ exists on $[0, \infty\rangle$ and it is continuous process, $\left(T_{M}, M \in \mathbb{N}\right)$ is an increasing sequence of stopping times such that $T_{M} \uparrow$ $+\infty$ a.s., when $M \rightarrow+\infty$. Let $\left(\Phi_{M}, M \in \mathbb{N}\right)$ be a sequence of $C^{\infty}(E)$ functions such that $\Phi_{M}=1$ on $C l\left(E_{M}\right)$, and $\Phi_{m}=0$ on $C l\left(E_{M+1}\right)^{c}$. Let us define the functions $\mu_{M}(x, \theta):=\Phi_{M}(x) \mu(x, \theta),(x, \theta) \in E \times \Theta$ and let $\nu_{M}$ be continuous functions on $E$ such that $\nu_{M}(x)=\nu(x)$ for $x \in C l\left(E_{M}\right)$ and $\nu_{M}(x)=$ const for $x \in E \backslash C l\left(E_{M+1}\right)$ (for example, $\nu_{M}$ can be defined as $\nu_{M}(x)=\Phi_{M}(x) \nu(x)+\left(1-\Phi_{M}(x)\right) c_{M}$, where $\left.c_{M}:=\min _{x \in C l\left(E_{M+1}\right)} \nu(x)\right)$. 
The functions $\mu_{M}, \nu_{M}$ are bounded and satisfy assumptions (A2) and (A3). Let, for a fixed $M \in \mathbb{N}$, the process $X^{M}=\left(X_{t}^{M}, 0 \leq t \leq T\right)$ be a unique strong solution of the SDE (see [17])

$$
d X_{t}^{M}=\mu_{M}\left(X_{t}^{M}, \theta_{0}\right) d t+\sigma_{0} \nu_{M}\left(X_{t}^{M}\right) d W_{t}, \quad X_{0}^{M}=x_{0}, x_{0} \in E .
$$

Moreover, for almost all $\omega \in \Omega$ and all $t \in\left[0, T_{M}(\omega)\right]$, it holds $X_{t}(\omega)=$ $\left(X^{M}\right)_{t}(\omega)$. Let $Y(\theta)$ be a random vector such that $Y(\theta)=\sqrt{\Sigma(\theta)} Z$, where $Z$ is standard normal random vector independent of $\mathcal{F}_{T}$. Let $Y_{M}$ be a random vector such that $Y_{M}(\theta)=\sqrt{\Sigma_{M}(\theta)} Z$, where $\Sigma_{M}(\theta)$ is a random matrix $\Sigma(\theta)$ from first part of this proof which we apply on process $X^{M}$ and functions $\nu_{M}$ and $\mu_{M}$. Let $\frac{1}{\sqrt{\Delta_{n}}}\left(D L_{T, M}(\theta)-D L_{n, M}(\theta)\right)$ be the left hand side of (5.2) for the process $X^{M}$, and the functions $\nu_{M}$ and $\mu_{M}$. Then, from first part of the proof $\frac{1}{\sqrt{\Delta_{n}}}\left(D L_{T, M}(\theta)-D L_{n, M}(\theta)\right) \stackrel{s t}{\Rightarrow} Y_{M}(\theta)$. With notation $Z_{n, M}(\theta):=$ $\frac{1}{\sqrt{\Delta_{n}}}\left(D L_{T, M}(\theta)-D L_{n, M}(\theta)\right)$ we can write $Z_{n, M}(\theta) \stackrel{s t}{\Rightarrow} Y_{M}(\theta)$. Let us denote by $Z_{n}(\theta):=\frac{1}{\sqrt{\Delta_{n}}}\left(D L_{T}(\theta)-D L_{n}(\theta)\right)$. We want to show that $Z_{n}(\theta) \stackrel{s t}{\Rightarrow} Y(\theta)$, for fixed $\theta \in \Theta$.

Let $f: \mathbb{R}^{d} \rightarrow \mathbb{R}$ be bounded continuous function, and let $U$ be bounded $\mathcal{F}_{T}$-measurable random variable. Let $B, H>0$ be constants such that $|f| \leq H$ and $|U| \leq B$. Now, we have:

$$
\begin{aligned}
& \left|\mathbb{E}\left[f\left(Z_{n}(\theta)\right) U\right]-\tilde{\mathbb{E}}[f(Y(\theta)) U]\right| \\
& \quad \leq\left|\mathbb{E}\left[f\left(Z_{n, M}(\theta)\right) U 1_{\left\{T_{M}>T\right\}}\right]-\tilde{\mathbb{E}}\left[f\left(Y_{M}(\theta)\right) U 1_{\left\{T_{M}>T\right\}}\right]\right|+2 B H \mathbb{P}\left(T_{M} \leq T\right) .
\end{aligned}
$$

$U 1_{\left\{T_{M}>T\right\}}$ is bounded, $\mathcal{F}_{T}$-measurable random variable, and $Z_{n, M}(\theta) \stackrel{s t}{\Rightarrow}$ $Y_{M}(\theta)$, hence

$$
\varlimsup_{n}\left|\mathbb{E}\left[f\left(Z_{n}(\theta)\right) U\right]-\tilde{\mathbb{E}}[f(Y(\theta)) U]\right| \leq 2 B H \mathbb{P}\left(T_{M} \leq T\right),
$$

and by letting $M \rightarrow \infty$ we have

$$
\varlimsup_{n}\left|\mathbb{E}\left[f\left(Z_{n}(\theta)\right) U\right]-\tilde{\mathbb{E}}[f(Y(\theta)) U]\right|=0,
$$

which implies

$$
\lim _{n}\left|\mathbb{E}\left[f\left(Z_{n}(\theta)\right) U\right]-\tilde{\mathbb{E}}[f(Y(\theta)) U]\right|=0,
$$

that proves our statement.

Theorem 5.4. Assume that (A1)-(A4) hold. Then

$$
\frac{1}{\sqrt{\Delta_{n}}}\left(D L_{T}(\hat{\theta})-D L_{n}(\hat{\theta})\right) \stackrel{s t}{\Rightarrow} Y(\hat{\theta}),
$$

where $Y(\hat{\theta}) \sim M N(0, \Sigma(\hat{\theta}))$. 
REMARK 5.5. From the proofs of Lemma 5.2 and Theorem 5.3 the following holds. If $X$ is a diffusion linear in drift parameters then the statement of Lemma 5.2 holds directly with $\theta$ replaced with $\hat{\theta}$. In that case the double-Itô integral term from the lemma does not depend on $\theta$. Hence the statement of Theorem 5.4 can be proved directly by following the proof of Theorem 5.3 and so without need for the additional assumption about relatively compactness of $\Theta$. Consequently $Y(\theta)$ does not depend on $\theta$.

Proof. Let $Z_{n}(\theta) \equiv Z_{n}(\omega, \theta):=\frac{1}{\sqrt{\Delta_{n}}}\left(D L_{T}(\omega, \theta)-D L_{n}(\omega, \theta)\right)$. Since $\hat{\theta}$ is $\mathcal{F}_{T^{-}}$measurable random vector, see [9, Lema 4.1.], and the functions $(\omega, \theta) \mapsto Z_{n} \equiv Z_{n}(\omega, \theta)$ are $\mathcal{F}_{T} \otimes \mathcal{B}(\Theta)$-measurable (see the beginning of the proof of $\left[9\right.$, Lemma 4.2.]), for each $n$, then $\omega \mapsto Z_{n}(\hat{\theta}) \equiv Z_{n}(\omega, \hat{\theta}(\omega))$ are $\mathcal{F}_{T}$-measurable functions. We want to prove that

$$
Z_{n}(\hat{\theta}) \stackrel{s t}{\Rightarrow} Y(\hat{\theta}) .
$$

First, let us assume that all of the functions which appear in the proof are bounded. Let $t \in \mathbb{R}^{d}$ be arbitrary, fixed, vector, and let $U$ be bounded $\mathcal{F}_{T^{-}}$ measurable random variable. Let $B>0$ be a constant such that $|U| \leq B$. For each $n \in \mathbb{N}$, let us define the functions $F_{n}$ in the following way:

$$
F_{n}(\omega, \theta):=\cos \left(\left\langle t, Z_{n}(\omega, \theta)\right\rangle\right)+i \sin \left(\left\langle t, Z_{n}(\omega, \theta)\right\rangle\right)-e^{-\frac{1}{2} \sum_{i, j=1}^{d} t_{i} t_{j} \Sigma(\omega, \theta)^{i j}} .
$$

For all $\omega \in \Omega$, the functions $\theta \mapsto F_{n}(\theta) \equiv F_{n}(\omega, \theta)$ are continuously differentiable on $\Theta$, and hence there exist nonnegative random variable $G_{n}$ and constant $R \geq 0$ (not depending on $n$ ) such that $\mathbb{E}\left[G_{n}\right] \leq R$ and

$$
\left|F_{n}\left(\theta_{1}\right)-F_{n}\left(\theta_{2}\right)\right| \leq G_{n}\left\|\theta_{1}-\theta_{2}\right\|, \quad \forall n \in \mathbb{N}, \forall \theta_{1}, \theta_{2} \in \Theta .
$$

Namely, since the rationals from $\Theta \cap \mathbb{Q}^{d}$ are dense in $\Theta$ and for $i=1, \ldots, d$, $\left|\partial \theta_{i} F_{n}(\theta)\right|$ are continuous on compact $C l(\Theta)$,

$$
G_{n}:=\sup _{\theta \in \Theta} \sum_{i=1}^{d}\left|\partial \theta_{i} F_{n}(\theta)\right|=\sup _{\theta \in \Theta \cap \mathbb{Q}^{d}} \sum_{i=1}^{d}\left|\partial \theta_{i} F_{n}(\theta)\right|
$$

is bounded and measurable, i.e. random variable.

By $[1$, Proposition 1] it is enough to prove that

$$
\lim _{n}\left|\mathbb{E}\left[F_{n}(\hat{\theta}) U\right]\right|=0 .
$$

Let $\epsilon>0$ be arbitrary, fixed real number. Define $\delta:=\frac{\epsilon}{2 R B}$. For all $\theta \in \Theta$, let $K(\theta, \delta)$ be a open ball in $\mathbb{R}^{d}$ with center $\theta$ and radius $\delta$. Since $C l(\Theta)$ is compact set and $\Theta \subseteq C l(\Theta) \subseteq \bigcup_{\theta \in \Theta} K(\theta, \delta)$, there exist finite number of balls $K\left(\theta_{l}, \delta\right), l=1, \ldots, N$ such that $\Theta \subseteq \bigcup_{l=1}^{N} K\left(\theta_{l}, \delta\right)$ and $\theta_{l} \in \Theta, l=1, \ldots, N$. 
Let us define a finite partition $\left\{K_{1}, \ldots, K_{N}\right\}$ of $\Theta$ in the following way:

$$
\begin{aligned}
K_{1} & :=K\left(\theta_{1}, \delta\right) \cap \Theta \\
K_{2} & :=K\left(\theta_{2}, \delta\right) \cap \Theta \cap K_{1}^{c} \\
& \vdots \\
K_{N} & :=K\left(\theta_{N}, \delta\right) \cap \Theta \cap K_{1}^{c} \cap K_{2}^{c} \cap \cdots \cap K_{N-1}^{c} .
\end{aligned}
$$

This partition does not depend on subdivision of the segment $[0, T]$. Since $1=1_{\{\hat{\theta} \in \Theta\}}=\sum_{l=1}^{N} 1_{\left\{\hat{\theta} \in K_{l}\right\}}$, we have

$$
\begin{aligned}
\left|\mathbb{E}\left[F_{n}(\hat{\theta}) U\right]\right| & =\left|\sum_{l=1}^{N} \mathbb{E}\left[F_{n}(\hat{\theta}) U 1_{\left\{\hat{\theta} \in K_{l}\right\}}\right]\right|= \\
& =\left|\sum_{l=1}^{N} \mathbb{E}\left[\left(F_{n}(\hat{\theta})-F_{n}\left(\theta_{l}\right)\right) U 1_{\left\{\hat{\theta} \in K_{l}\right\}}\right]+\sum_{l=1}^{N} \mathbb{E}\left[F_{n}\left(\theta_{l}\right) U 1_{\left\{\hat{\theta} \in K_{l}\right\}}\right]\right| \\
& \leq\left|\sum_{l=1}^{N} \mathbb{E}\left[\left(F_{n}(\hat{\theta})-F_{n}\left(\theta_{l}\right)\right) U 1_{\left\{\hat{\theta} \in K_{l}\right\}}\right]\right|+\left|\sum_{l=1}^{N} \mathbb{E}\left[F_{n}\left(\theta_{l}\right) U 1_{\left\{\hat{\theta} \in K_{l}\right\}}\right]\right| .
\end{aligned}
$$

Events $\left\{\left\{\hat{\theta} \in K_{1}\right\}, \ldots,\left\{\hat{\theta} \in K_{N}\right\}\right\}$ make complete system of events for $\Theta$. On the event $\left\{\hat{\theta} \in K_{l}\right\}$ holds $\left\|\hat{\theta}-\theta_{l}\right\|<\delta$, so from (5.12) it follows

$$
\begin{aligned}
& \left|\sum_{l=1}^{N} \mathbb{E}\left[\left(F_{n}(\hat{\theta})-F_{n}\left(\theta_{l}\right)\right) U 1_{\left\{\hat{\theta} \in K_{l}\right\}}\right]\right| \leq \sum_{l=1}^{N} \mathbb{E}\left[\left|F_{n}(\hat{\theta})-F_{n}\left(\theta_{l}\right) \| U\right| 1_{\left\{\hat{\theta} \in K_{l}\right\}}\right] \\
& \quad \leq B \sum_{l=1}^{N} \mathbb{E}\left[G_{n}\left\|\hat{\theta}-\theta_{l}\right\| 1_{\left\{\hat{\theta} \in K_{l}\right\}}\right]<B \delta \sum_{l=1}^{N} \mathbb{E}\left[G_{n} 1_{\left\{\hat{\theta} \in K_{l}\right\}}\right] \\
& \quad=B \delta \mathbb{E}\left[G_{n}\right] \leq B R \delta=\frac{\epsilon}{2}
\end{aligned}
$$

Since $\hat{\theta}$ is $\mathcal{F}_{T}$ - measurable random vector, it implies that $U 1_{\left\{\hat{\theta} \in K_{l}\right\}}$ is bounded, $\mathcal{F}_{T}$-measurable random variable, $l=1, \ldots, N$. Theorem 5.3 and $[1$, Proposition 1] implies

$$
\lim _{n} \mathbb{E}\left[F_{n}\left(\theta_{l}\right) U 1_{\left\{\hat{\theta} \in K_{l}\right\}}\right]=0, \quad l=1, \ldots, N,
$$

which yields

$$
\lim _{n}\left|\sum_{l=1}^{N} \mathbb{E}\left[F_{n}\left(\theta_{l}\right) U 1_{\left\{\tilde{\theta} \in K_{l}\right\}}\right]\right|=0 .
$$

Let $n_{0}=n_{0}(\epsilon) \in \mathbb{N}$ be natural number such that for all $n \geq n_{0}$

$$
\left|\sum_{l=1}^{N} \mathbb{E}\left[F_{n}\left(\theta_{l}\right) U 1_{\left\{\hat{\theta} \in K_{l}\right\}}\right]\right|<\frac{\epsilon}{2} .
$$


For (5.13) we have

$$
\begin{aligned}
\left|\mathbb{E}\left[F_{n}(\hat{\theta}) U\right]\right| & \leq\left|\sum_{l=1}^{N} \mathbb{E}\left[\left(F_{n}(\hat{\theta})-F_{n}\left(\theta_{l}\right)\right) U 1_{\left.\hat{\theta} \in K_{l}\right\}}\right]\right|+\left|\sum_{l=1}^{N} \mathbb{E}\left[F_{n}\left(\theta_{l}\right) U 1_{\left\{\hat{\theta} \in K_{l}\right\}}\right]\right| \\
& \leq \frac{\epsilon}{2}+\frac{\epsilon}{2}=\epsilon, \quad \forall n \geq n_{0},
\end{aligned}
$$

so the theorem holds for bounded functions. In the general case, let us define stopping times $T_{M}$ as in the proof of Theorem 5.3. Let $\hat{\theta}_{M}$ be a solution of the equation $D L_{T, M}(\theta)=0$. Since $\hat{\theta}$ is a unique solution of $D L_{T}(\theta)=0$, and $D L_{T, M}=D L_{T}$ on $\left\{T_{M}>T\right\} \times \Theta$, it implies that $\hat{\theta}_{M}$ and $\hat{\theta}$ coincide on $\left\{T_{M}>T\right\}$. In the first part of the proof we proved $\frac{1}{\sqrt{\Delta_{n}}}\left(D L_{T, M}\left(\hat{\theta}_{M}\right)-\right.$ $\left.D L_{n, M}\left(\hat{\theta}_{M}\right)\right) \stackrel{s t}{\Rightarrow} Y_{M}\left(\hat{\theta}_{M}\right)$, shortly $Z_{n, M}\left(\hat{\theta}_{M}\right) \stackrel{s t}{\Rightarrow} Y_{M}\left(\hat{\theta}_{M}\right)$. Let $f: \mathbb{R}^{d} \rightarrow \mathbb{R}$ be bounded continuous function and let $H>0$ be a real number such that $|f(x)| \leq H, \forall x \in \mathbb{R}^{d}$.

$$
\begin{aligned}
& \left|\mathbb{E}\left[f\left(Z_{n}(\hat{\theta})\right) U\right]-\tilde{\mathbb{E}}[f(Y(\hat{\theta})) U]\right| \\
& \quad \leq\left|\mathbb{E}\left[f\left(Z_{n, M}\left(\hat{\theta}_{M}\right)\right) U 1_{\left\{T_{M}>T\right\}}\right]-\tilde{\mathbb{E}}\left[f\left(Y_{M}\left(\hat{\theta}_{M}\right)\right) U 1_{\left\{T_{M}>T\right\}}\right]\right| \\
& \quad+2 B H \mathbb{P}\left(T_{M} \leq T\right) .
\end{aligned}
$$

Since $U 1_{\left\{T_{M}>T\right\}}$ is bounded, $\mathcal{F}_{T}$-measurable random variable and $Z_{n, M}\left(\hat{\theta}_{M}\right)$ $\stackrel{s t}{\Rightarrow} Y_{M}\left(\hat{\theta}_{M}\right)$, we have

$$
\varlimsup_{n}\left|\mathbb{E}\left[f\left(Z_{n}(\hat{\theta})\right) U\right]-\tilde{\mathbb{E}}[f(Y(\hat{\theta})) U]\right| \leq 2 B H \mathbb{P}\left(T_{M} \leq T\right),
$$

and by letting $M \rightarrow \infty$, we get

$$
\varlimsup_{n}\left|\mathbb{E}\left[f\left(Z_{n}(\hat{\theta})\right) U\right]-\tilde{\mathbb{E}}[f(Y(\hat{\theta})) U]\right|=0,
$$

which implies

$$
\lim _{n}\left|\mathbb{E}\left[f\left(Z_{n}(\hat{\theta})\right) U\right]-\tilde{\mathbb{E}}[f(Y(\hat{\theta})) U]\right|=0
$$

Lemma 5.6. Assume that (A1)-(A4) hold. Assume that $\left(\bar{\theta}_{n}, n \in \mathbb{N}\right)$ satisfies (4.2)-(4.5). Then

$$
A_{\hat{\theta}, \bar{\theta}_{n}} \frac{1}{\sqrt{\Delta_{n}}}\left(\bar{\theta}_{n}-\hat{\theta}\right) \stackrel{s t}{\Rightarrow} Y(\hat{\theta})
$$

where $Y(\hat{\theta}) \sim M N(0, \Sigma(\hat{\theta}))$.

Proof. First, let $\nu^{-1}$ be a bounded function and let $\mu, \nu$ and all of the functions which appear in the proof be bounded. Since (4.2) holds, it implies 
that $1_{\left\{D L_{n}\left(\bar{\theta}_{n}\right)=0\right\}} \stackrel{\mathbb{P}}{\rightarrow} 1$ and $1_{\left\{D L_{n}\left(\bar{\theta}_{n}\right) \neq 0\right\}} \stackrel{\mathbb{P}}{\rightarrow} 0$. For $A_{\hat{\theta}, \bar{\theta}_{n}} \frac{1}{\sqrt{\Delta_{n}}}\left(\bar{\theta}_{n}-\hat{\theta}\right)$ holds

$$
\begin{aligned}
A_{\hat{\theta}, \bar{\theta}_{n}} & \frac{1}{\sqrt{\Delta_{n}}}\left(\bar{\theta}_{n}-\hat{\theta}\right)= \\
= & \frac{1}{\sqrt{\Delta_{n}}}\left(D L_{T}(\hat{\theta})-D L_{n}(\hat{\theta})\right) 1_{\left\{D L_{n}\left(\bar{\theta}_{n}\right)=0\right\}} \\
& +A_{\hat{\theta}, \bar{\theta}_{n}} \frac{1}{\sqrt{\Delta_{n}}}\left(\bar{\theta}_{n}-\hat{\theta}\right) 1_{\left\{D L_{n}\left(\bar{\theta}_{n}\right) \neq 0\right\}} .
\end{aligned}
$$

Theorem 5.4 and $[12,(2.2 .5)]$ imply

$$
\left(\frac{1}{\sqrt{\Delta_{n}}}\left(D L_{T}(\hat{\theta})-D L_{n}(\hat{\theta})\right), 1_{\left\{D L_{n}\left(\bar{\theta}_{n}\right)=0\right\}}\right) \stackrel{s t}{\Rightarrow}(Y(\hat{\theta}), 1)
$$

hence from [1, Theorem 1] follows that

$$
\frac{1}{\sqrt{\Delta_{n}}}\left(D L_{T}(\hat{\theta})-D L_{n}(\hat{\theta})\right) 1_{\left\{D L_{n}\left(\bar{\theta}_{n}\right)=0\right\}} \stackrel{s t}{\Rightarrow} Y(\hat{\theta}) .
$$

It holds

$$
\left\|A_{\hat{\theta}, \bar{\theta}_{n}} \frac{1}{\sqrt{\Delta_{n}}}\left(\bar{\theta}_{n}-\hat{\theta}\right) 1_{\left\{D L_{n}\left(\bar{\theta}_{n}\right) \neq 0\right\}}\right\| \leq\left\|A_{\hat{\theta}, \bar{\theta}_{n}}\right\| \frac{1}{\sqrt{\Delta_{n}}}\left\|\bar{\theta}_{n}-\hat{\theta}\right\| 1_{\left\{D L_{n}\left(\bar{\theta}_{n}\right) \neq 0\right\}} .
$$

$1_{\left\{D L_{n}\left(\bar{\theta}_{n}\right) \neq 0\right\}} \stackrel{\mathbb{P}}{\rightarrow} 0$ and sequences $\left\|A_{\hat{\theta}, \bar{\theta}_{n}}\right\|$ and $\frac{1}{\sqrt{\Delta_{n}}}\left\|\bar{\theta}_{n}-\hat{\theta}\right\|$ are bounded in probability, so we can conclude that $A_{\hat{\theta}, \bar{\theta}_{n}} \frac{1}{\sqrt{\Delta_{n}}}\left(\bar{\theta}_{n}-\hat{\theta}\right) 1_{\left\{D L_{n}\left(\bar{\theta}_{n}\right) \neq 0\right\}} \stackrel{\mathbb{P}}{\rightarrow} 0$, hence by $[11,(2.2)]$ this theorem holds for bounded functions. For general case, we define a sequence of stopping times $T_{M}$ (as in the proof of Theorem 5.4). Let $\hat{\theta}_{M}$ be defined in the same way as in Theorem 5.4. Let $\bar{\theta}_{n, M}$ be a sequence which satisfies (4.2)-(4.5) for the functions $\mu_{M}, \nu_{M}$ and the process $X^{M}$. Since (4.4) holds, we have $\lim _{n} \mathbb{P}\left(\bar{\theta}_{n, M}=\bar{\theta}_{n} \mid T_{M}>T\right)=1$. Let $f$ be bounded real function, and $U$ be a bounded random variable. Let $B, H>0$ be a constants such that $|U| \leq B$ and $|f| \leq H$. Then, with definition $A_{\theta_{1}, \theta_{2}, M}:=\int_{0}^{1} D^{2} L_{n, M}\left(\theta_{1}+v\left(\theta_{2}-\theta_{1}\right)\right) d v$, we have

$$
\begin{aligned}
\left|\mathbb{E}\left[f\left(A_{\hat{\theta}, \bar{\theta}_{n}} \frac{1}{\sqrt{\Delta_{n}}}\left(\bar{\theta}_{n}-\hat{\theta}\right)\right) U\right]-\tilde{\mathbb{E}}[f(Y(\hat{\theta})) U]\right| \\
\leq \mid \mathbb{E}\left[f\left(A_{\hat{\theta}_{,}, \bar{\theta}_{n}, M} \frac{1}{\sqrt{\Delta_{n}}}\left(\bar{\theta}_{n}-\hat{\theta}\right)\right) U 1_{\left\{T_{M}>T\right\}} 1_{\left\{\bar{\theta}_{n, M}=\bar{\theta}_{n}\right\}}\right] \\
\quad-\tilde{\mathbb{E}}\left[f\left(Y_{M}\left(\hat{\theta}_{M}\right)\right) U 1_{\left\{T_{M}>T\right\}}\right] \mid+2 B H \mathbb{P}\left(T_{M} \leq T\right) \\
\quad+B H \mathbb{P}\left(\bar{\theta}_{n, M} \neq \bar{\theta}_{n}, T_{M}>T\right)
\end{aligned}
$$

and the proof is over once we let first $n \rightarrow+\infty$, and then $M \rightarrow+\infty$. 
Lemma 5.7. Assume that (A1)-(A4) hold. Assume that $\left(\bar{\theta}_{n}, n \in \mathbb{N}\right)$ satisfies (4.2)-(4.5). Then

$$
\int_{0}^{1} D^{2} L_{T}(\hat{\theta})-D^{2} L_{T}\left(\hat{\theta}+v\left(\bar{\theta}_{n}-\hat{\theta}\right)\right) d v \stackrel{\mathbb{P}}{\rightarrow} 0 .
$$

Proof. It is sufficient to prove that $\sup _{v \in[0,1]} \| D^{2} L_{T}(\hat{\theta})-D^{2} L_{T}(\hat{\theta}+$ $\left.v\left(\bar{\theta}_{n}-\hat{\theta}\right)\right) \| \stackrel{\mathbb{P}}{\rightarrow} 0$. We will prove that $\sup _{v \in[0,1]} F\left(\bar{\theta}_{n}, v\right) \stackrel{\mathbb{P}}{\rightarrow} 0$, for the functions

$$
F(\theta, v) \equiv F(\omega, \theta, v):=\left\|D^{2} L_{T}(\omega, \hat{\theta}(\omega))-D^{2} L_{T}(\omega, \hat{\theta}(\omega)+v(\theta-\hat{\theta}(\omega)))\right\| .
$$

For details see the Appendix.

Lemma 5.8. Assume that (A1)-(A4) hold. Assume that $\left(\bar{\theta}_{n}, n \in \mathbb{N}\right)$ satisfies (4.2)-(4.5). Then

$$
D^{2} L_{T}(\hat{\theta}) \frac{1}{\sqrt{\Delta_{n}}}\left(\bar{\theta}_{n}-\hat{\theta}\right) \stackrel{s t}{\Rightarrow} M N(0, \Sigma(\hat{\theta}))
$$

Proof. We will prove that the sequence $D^{2} L_{T}(\hat{\theta}) \frac{1}{\sqrt{\Delta_{n}}}\left(\bar{\theta}_{n}-\hat{\theta}\right)$ can be written as the sum of the sequence which converges in probability to zero and the sequence which converges stably in law to $M N(0, \Sigma(\hat{\theta}))$. For details see the Appendix.

Lemma 5.9. Assume that (A1)-(A4) hold. Assume that $\left(\bar{\theta}_{n}, n \in \mathbb{N}\right)$ satisfies (4.2)-(4.5). Then

$$
D^{2} L_{n}\left(\bar{\theta}_{n}\right) \frac{1}{\sqrt{\Delta_{n}}}\left(\bar{\theta}_{n}-\hat{\theta}\right) \stackrel{s t}{\Rightarrow} Y(\hat{\theta})
$$

PROOF.

$$
\begin{aligned}
D^{2} L_{n}\left(\bar{\theta}_{n}\right) \frac{1}{\sqrt{\Delta_{n}}}\left(\bar{\theta}_{n}-\hat{\theta}\right) \\
=\left(D^{2} L_{n}\left(\bar{\theta}_{n}\right)-D^{2} L_{T}\left(\bar{\theta}_{n}\right)\right) \frac{1}{\sqrt{\Delta_{n}}}\left(\bar{\theta}_{n}-\hat{\theta}\right) \\
\quad+\left(D^{2} L_{T}\left(\bar{\theta}_{n}\right)-D^{2} L_{T}(\hat{\theta})\right) \frac{1}{\sqrt{\Delta_{n}}}\left(\bar{\theta}_{n}-\hat{\theta}\right)+D^{2} L_{T}(\hat{\theta}) \frac{1}{\sqrt{\Delta_{n}}}\left(\bar{\theta}_{n}-\hat{\theta}\right) .
\end{aligned}
$$

(4.6) implies

$$
D^{2} L_{n}\left(\bar{\theta}_{n}\right)-D^{2} L_{T}\left(\bar{\theta}_{n}\right) \stackrel{\mathbb{P}}{\rightarrow} 0,
$$

and since the sequence $\frac{1}{\sqrt{\Delta_{n}}}\left(\bar{\theta}_{n}-\hat{\theta}\right)$ is bounded in probability, it follows that

$$
\left(D^{2} L_{n}\left(\bar{\theta}_{n}\right)-D^{2} L_{T}\left(\bar{\theta}_{n}\right)\right) \frac{1}{\sqrt{\Delta_{n}}}\left(\bar{\theta}_{n}-\hat{\theta}\right) \stackrel{\mathbb{P}}{\rightarrow} 0 .
$$

From Lemma 5.7, when $v=1$, we get

$$
D^{2} L_{T}\left(\bar{\theta}_{n}\right)-D^{2} L_{T}(\hat{\theta}) \stackrel{\mathbb{P}}{\rightarrow} 0
$$


hence

$$
\left(D^{2} L_{T}\left(\bar{\theta}_{n}\right)-D^{2} L_{T}(\hat{\theta})\right) \frac{1}{\sqrt{\Delta_{n}}}\left(\bar{\theta}_{n}-\hat{\theta}\right) \stackrel{\mathbb{P}}{\rightarrow} 0 .
$$

Lemma 5.8 and $[11,(2.2)]$ imply the conclusion.

Lemma 5.10. Assume that (A1)-(A5) hold. Assume that $\left(\bar{\theta}_{n}, n \in \mathbb{N}\right)$ satisfies (4.2)-(4.5). Then

$$
(\sqrt{\Sigma(\hat{\theta})})^{-1} D^{2} L_{n}\left(\bar{\theta}_{n}\right) \frac{1}{\sqrt{\Delta_{n}}}\left(\bar{\theta}_{n}-\hat{\theta}\right) \stackrel{s t}{\Rightarrow} N(0, I) .
$$

Proof. This Lemma is consequence of Lemma 5.9 and [1, Theorem 1].

Lemma 5.11. Assume that (A1)-(A4) hold. Assume that $\left(\bar{\theta}_{n}, n \in \mathbb{N}\right)$ satisfies (4.2)-(4.5). Then

$$
\Sigma_{n}\left(\bar{\theta}_{n}\right) \stackrel{\mathbb{P}}{\rightarrow} \Sigma(\hat{\theta}) .
$$

Proof. We will prove that each component $\Sigma_{n}\left(\bar{\theta}_{n}\right)^{j k}$ can be written as the sum of sequence which converges in probability to zero and the random variable $\Sigma(\hat{\theta})^{j k}$. For details see the Appendix.

Lemma 5.12. Assume that (A1)-(A5) hold. Assume that $\left(\bar{\theta}_{n}, n \in \mathbb{N}\right)$ satisfies (4.2)-(4.5). Then

$$
\lim _{n \rightarrow \infty} \mathbb{P}\left(\Sigma_{n}\left(\bar{\theta}_{n}\right) \text { is regular matrix }\right)=1 .
$$

Proof. For details see the Appendix.

\subsection{Proofs of Theorem 4.1 and Corollary 4.2.}

Proof of Theorem 4.1. Since $D^{2} L_{T}(\hat{\theta})$ is regular symmetric matrix, there exists $\left(D^{2} L_{T}(\hat{\theta})\right)^{-1}$, and it is symmetric matrix. Since $D^{2} L_{T}(\hat{\theta})$ is $\mathcal{F}_{T}$-measurable random matrix, and multiplication with inverse is continuous mapping, [1, Theorem 1] and Lemma 5.8 imply

$$
\frac{1}{\sqrt{\Delta_{n}}}\left(\bar{\theta}_{n}-\hat{\theta}\right) \stackrel{s t}{\Rightarrow}\left(D^{2} L_{T}(\hat{\theta})\right)^{-1} M N(0, \Sigma(\hat{\theta})),
$$

where $\left(D^{2} L_{T}(\hat{\theta})\right)^{-1} M N(0, \Sigma(\hat{\theta})) \sim M N\left(0,\left(D^{2} L_{T}(\hat{\theta})\right)^{-1} \Sigma(\hat{\theta})\left(D^{2} L_{T}(\hat{\theta})\right)^{-1}\right)$.

Proof of Corollary 4.2. Using continuous mapping theorem, from Lemma 5.11 and Lemma 5.12 it follows that

$$
\left(\sqrt{\Sigma_{n}\left(\bar{\theta}_{n}\right)}\right)^{-1} 1_{\left\{\Sigma_{n}\left(\bar{\theta}_{n}\right) \text { is regular matrix }\right\}} \stackrel{\mathbb{P}}{\rightarrow}(\sqrt{\Sigma(\hat{\theta})})^{-1}, \quad n \rightarrow \infty .
$$




$$
\begin{aligned}
& \text { For }\left(\sqrt{\Sigma_{n}\left(\bar{\theta}_{n}\right)}\right)^{-1} D^{2} L_{n}\left(\bar{\theta}_{n}\right) \frac{1}{\sqrt{\Delta_{n}}}\left(\bar{\theta}_{n}-\hat{\theta}\right) 1_{\left\{\Sigma_{n}\left(\bar{\theta}_{n}\right) \text { is regular matrix }\right\}} \text { one may write } \\
& \qquad \begin{aligned}
\left(\sqrt{\Sigma_{n}\left(\bar{\theta}_{n}\right)}\right)^{-1} D^{2} L_{n}\left(\bar{\theta}_{n}\right) \frac{1}{\sqrt{\Delta_{n}}}\left(\bar{\theta}_{n}-\hat{\theta}\right) 1_{\left\{\Sigma_{n}\left(\bar{\theta}_{n}\right) \text { is regular matrix }\right\}} \\
=\left(\left(\sqrt{\Sigma_{n}\left(\bar{\theta}_{n}\right)}\right)^{-1} 1_{\left\{\Sigma_{n}\left(\bar{\theta}_{n}\right) \text { is regular matrix }\right\}}\right. \\
\left.-(\sqrt{\Sigma(\hat{\theta})})^{-1}\right) D^{2} L_{n}\left(\bar{\theta}_{n}\right) \frac{1}{\sqrt{\Delta_{n}}}\left(\bar{\theta}_{n}-\hat{\theta}\right) \\
+(\sqrt{\Sigma(\hat{\theta})})^{-1} D^{2} L_{n}\left(\bar{\theta}_{n}\right) \frac{1}{\sqrt{\Delta_{n}}}\left(\bar{\theta}_{n}-\hat{\theta}\right) .
\end{aligned}
\end{aligned}
$$

Since $D^{2} L_{n}\left(\bar{\theta}_{n}\right) \frac{1}{\sqrt{\Delta_{n}}}\left(\bar{\theta}_{n}-\hat{\theta}\right), n \in \mathbb{N}$ converges stably in law (Lemma 5.9), it also converges in distribution, which implies that this sequence is bounded in probability. Hence, it follows that

$$
\begin{aligned}
& \left(\left(\sqrt{\Sigma_{n}\left(\bar{\theta}_{n}\right)}\right)^{-1} 1_{\left\{\Sigma_{n}\left(\bar{\theta}_{n}\right) \text { is regular matrix }\right\}}\right. \\
& \left.\quad-(\sqrt{\Sigma(\hat{\theta})})^{-1}\right) D^{2} L_{n}\left(\bar{\theta}_{n}\right) \frac{1}{\sqrt{\Delta_{n}}}\left(\bar{\theta}_{n}-\hat{\theta}\right) \stackrel{\mathbb{P}}{\rightarrow} 0
\end{aligned}
$$

and Lemma 5.10 and $[11,(2.2)]$ yields the result.

\section{Simulations}

EXAMPLE 6.1. SDE

$$
d X_{t}=a X_{t} d t+d W_{t}, \quad X_{0}=0,
$$

is given, where $a \in \mathbb{R}$ is unknown parameter, and process is observed over fixed time interval $[0, T]$. For $n \in \mathbb{N}$, define $h=\frac{T}{n}$ and equidistant subdivision of $[0, T]$ with $t_{i}=i h, i=1, \ldots, n$. Then MLE, based on the continuous-time sample $\left(X_{t}\right)_{t \in[0, T]}, \hat{a}$ is given by (see [16])

$$
\hat{a}=\frac{X_{T}^{2}-T}{2 \int_{0}^{T} X_{t}^{2} d t} .
$$

Let $\left(X_{1}, \ldots, X_{n}\right):=\left(X_{t_{1}}, \ldots, X_{t_{n}}\right)$ be discrete random sample from the model. For the simulation study, $\int_{0}^{T} X_{t}^{2} d t$ will be approximated by $h \sum_{i=1}^{n} X_{i-1}^{2}$, hence $\hat{a}$ will be estimated by the formula

$$
\hat{a}=\frac{X_{T}^{2}-T}{2 h \sum_{i=1}^{n} X_{i-1}^{2}} .
$$


If we define the functions $\mu(x, a)=a x, \nu(x)=1$, and $\sigma_{0}=1$, then using (3.3) we get

$$
L_{n}(a)=\sum_{i=1}^{n}\left(\left(X_{i}-X_{i-1}\right) X_{i-1} a-\frac{1}{2} X_{i-1}^{2} h a^{2}\right),
$$

from which we calculate AMLE $\bar{a}_{n}$, which is given by formula

$$
\bar{a}_{n}=\frac{\sum_{i=1}^{n}\left(X_{i}-X_{i-1}\right) X_{i-1}}{h \sum_{i=1}^{n} X_{i-1}^{2}} .
$$

Therefore, all of the assumptions of Corollary 4.2 are satisfied, and we have

$$
-\frac{\sqrt{2}}{\sqrt{n}} \sum_{i=1}^{n} X_{i-1}^{2}\left(\bar{a}_{n}-\hat{a}\right) \stackrel{s t}{\Rightarrow} N(0,1) .
$$

Let $-z_{\frac{\alpha}{2}}$ be $\frac{\alpha}{2}$-quantile of standard normal distribution. We simulated $M$ realizations of discrete random sample $\left(X_{t_{1}}, \ldots, X_{t_{n}}\right)$ with parameter $a=2$ over equidistant points $t_{i}=i \Delta_{n}$, where $n=2^{k}, T=1$ and $\Delta_{n}=\frac{T}{n}$. Then we calculated how many times values of the variable

$$
-\frac{\sqrt{2}}{\sqrt{n}} \sum_{i=1}^{n} X_{i-1}^{2}\left(\bar{a}_{n}-\hat{a}\right)
$$

are in the interval $\left[-z_{\frac{\alpha}{2}}, z_{\frac{\alpha}{2}}\right]$ and we present that number as a percentage. Results are presented in Tables 1, 2 and 3. Simulations showed (see Tables 1, 2

TABLE 1. $M=100, \alpha=0.05$

\begin{tabular}{|l|llllllll|}
\hline$k$ & 2 & 4 & 6 & 8 & 10 & 12 & 14 & 16 \\
\hline$\%$ & 0.51 & 0.55 & 0.82 & 0.84 & 0.90 & 0.97 & 0.95 & 0.96 \\
\hline
\end{tabular}

TABLE 2. $M=100, \alpha=0.01$

\begin{tabular}{|l|llllllll|}
\hline$k$ & 2 & 4 & 6 & 8 & 10 & 12 & 14 & 16 \\
\hline$\%$ & 0.52 & 0.72 & 0.83 & 0.91 & 0.95 & 0.98 & 1 & 0.97 \\
\hline
\end{tabular}

TABLE 3. $M=1000, \alpha=0.05$

\begin{tabular}{|l|llllllll|}
\hline$k$ & 2 & 4 & 6 & 8 & 10 & 12 & 14 & 16 \\
\hline$\%$ & 0.541 & 0.670 & 0.825 & 0.918 & 0.976 & 0.989 & 0.987 & 0.988 \\
\hline
\end{tabular}

and 3) that increasing the number $n$ causes increase in percentage of values of variable (6.3) which are in the interval $\left[-z_{\frac{\alpha}{2}}, z_{\frac{\alpha}{2}}\right]$, in the sense that percentage becomes value near $(1-\alpha) \cdot 100 \%$, what was expected. 
EXAmPLE 6.2. Gompertz model is given by SDE

$$
d X_{t}=\left(a-b \ln X_{t}\right) X_{t} d t+\sigma_{0} X_{t} d W_{t}, X_{0}=1,
$$

where $a, b \in \mathbb{R}$ are unknown parameters, and $\sigma_{0}^{2}=0.84$. Process is observed over time-interval $[0, T]$. In this example, we define the functions $\mu(x, a, b)=$ $(a-b \ln x) x$ and $\nu(x)=x$. MLE $\hat{a}, \hat{b}$ of the parameters $a, b$ are calculated using [3, Equation 20] and given by

$$
\begin{aligned}
\hat{b} & =\frac{T \int_{0}^{T} \frac{\ln X_{s}}{X_{s}} d X_{s}-\int_{0}^{T} \frac{1}{X_{s}} d X_{s} \cdot \int_{0}^{T} \ln X_{s} d s}{\left(\int_{0}^{T} \ln X_{s} d s\right)^{2}-T \int_{0}^{T}\left(\ln X_{s}\right)^{2} d s}, \\
\hat{a} & =\frac{\hat{b} \int_{0}^{T} \ln X_{s} d s+\int_{0}^{T} \frac{1}{X_{s}} d X_{s}}{T} .
\end{aligned}
$$

If $\left(X_{1}, \ldots, X_{n}\right):=\left(X_{t_{1}}, \ldots, X_{t_{n}}\right)$ is discrete random sample from the model, then using $(3.3)$ we get

$$
L_{n}(a, b)=\sum_{i=1}^{n}\left(\frac{\left(X_{i}-X_{i-1}\right)\left(a-b \ln X_{i-1}\right)}{0.84 X_{i-1}}-\frac{1}{2} \frac{\left(a-b \ln X_{i-1}\right)^{2} h}{0.84}\right) .
$$

$L_{n}$ is quadratic function of $a$ and $b$, which means that there exists a point of maximum of that function. Hence, AMLE $\bar{a}_{n}$ and $\bar{b}_{n}$ of the parameters $a$ and $b$ can be calculated using formulas

$$
\begin{aligned}
\bar{b}_{n} & =\frac{\sum_{i=1}^{n} \frac{X_{i}-X_{i-1}}{X_{i-1}} \cdot \sum_{i=1}^{n} \ln X_{i-1}-n \sum_{i=1}^{n} \frac{X_{i}-X_{i-1}}{X_{i-1}} \ln X_{i-1}}{T \sum_{i=1}^{n}\left(\ln X_{i-1}\right)^{2}-h\left(\sum_{i=1}^{n} \ln X_{i-1}\right)^{2}}, \\
\bar{a}_{n} & =\frac{\bar{b}_{n} h \sum_{i=1}^{n} \ln X_{i-1}+\sum_{i=1}^{n} \frac{X_{i}-X_{i-1}}{X_{i-1}}}{T} .
\end{aligned}
$$

In this case MLE given by (6.4) can not be calculated exactly, so we will use formulas (6.5) as their good approximation. For that purpose we used different (much larger) number of points for calculating MLE then for AMLE. In this way obtained estimate of MLE is not equal to estimate of AMLE. If we define the functions $\mu(x, a, b)=a-b \ln x, \nu(x)=x$, then all of the assumptions of Corollary 4.2 are satisfied, and we have

$$
\left\|\left(\sqrt{\Sigma_{n}\left(\bar{\theta}_{n}\right)}\right)^{-1} D^{2} L_{n}\left(\bar{\theta}_{n}\right) \frac{1}{\sqrt{\Delta_{n}}}\left(\bar{\theta}_{n}-\hat{\theta}\right)\right\|^{2} \stackrel{s t}{\Rightarrow} \chi_{2}^{2},
$$

where $\theta=(a, b)$, and matrices $\Sigma_{n}\left(\bar{\theta}_{n}\right)$ and $D^{2} L_{n}\left(\bar{\theta}_{n}\right)$ are given by formulas

$$
\begin{gathered}
\Sigma_{n}\left(\bar{\theta}_{n}\right)=\left[\begin{array}{cc}
\frac{T}{2} & \frac{T-h \sum_{i=1}^{n} \ln X_{i-1}}{2} \\
\frac{T-h \sum_{i=1}^{n} \ln X_{i-1}}{2} & \frac{T-2 h \sum_{i=1}^{n} \ln X_{i-1}+h \sum_{i=1}^{n}\left(\ln X_{i-1}\right)^{2}}{2}
\end{array}\right], \\
D^{2} L_{n}\left(\bar{\theta}_{n}\right)=\frac{1}{\sigma_{0}^{2}}\left[\begin{array}{cc}
-T & h \sum_{i=1}^{n} \ln X_{i-1} \\
h \sum_{i=1}^{n} \ln X_{i-1} & -h \sum_{i=1}^{n}\left(\ln X_{i-1}\right)^{2}
\end{array}\right] .
\end{gathered}
$$


We simulated $M$ realizations of discrete random sample $\left(X_{t_{1}}, \ldots, X_{t_{n}}\right)$ with parameters $a=9.72, b=3.7$, over equidistant points $t_{i}=i \Delta_{n}$, where $n=2^{k}$, $T=1$ and $\Delta_{n}=\frac{T}{n}$. We simulated in the way that $n^{\prime}=2^{l}>2^{k}$ and we use all of these points to estimate MLE $\hat{\theta}$ by using (6.5). Then we take subsample of length $n=2^{k}$ and calculate AMLE $\bar{\theta}_{n}$ by (6.5). Then, we calculate percentage of values

$\left\langle\left(\sqrt{\Sigma_{n}\left(\bar{\theta}_{n}\right)}\right)^{-1} D^{2} L_{n}\left(\bar{\theta}_{n}\right) \frac{1}{\sqrt{\Delta_{n}}}\left(\bar{\theta}_{n}-\hat{\theta}\right),\left(\sqrt{\Sigma_{n}\left(\bar{\theta}_{n}\right)}\right)^{-1} D^{2} L_{n}\left(\bar{\theta}_{n}\right) \frac{1}{\sqrt{\Delta_{n}}}\left(\bar{\theta}_{n}-\hat{\theta}\right)\right\rangle$

which are in the interval $\left[0, \chi_{1-\alpha}^{2}\right]$, where $\chi_{1-\alpha}^{2}$ is $(1-\alpha)$-quantile of $\chi^{2}$ distribution with 2 degrees of freedom. Results are presented in Tables 4, 5, 6, 7 and 8 . Simulations showed (see Tables $6,7,8$ ) that increase in number $k$

TABLE $4 . \quad M=100, \alpha=0.025$

\begin{tabular}{|l|l|llllllllll|l|}
\cline { 2 - 13 } \multicolumn{1}{c|}{} & $l$ & 4 & 5 & 6 & 8 & 10 & 10 & 10 & 10 & 12 & 13 \\
& $k$ & 2 & 2 & 2 & 2 & 2 & 4 & 6 & 8 & 6 & 6 \\
\cline { 2 - 14 } \multicolumn{1}{c|}{} & $\%$ & 0.26 & 0.26 & 0.17 & 0.29 & 0.28 & 0.65 & 0.93 & 0.99 & 0.9 & 0.9 \\
\hline$l$ & 13 & 13 & 14 & 14 & 14 & 14 & 15 & 15 & 15 & 16 & 16 \\
$k$ & 7 & 8 & 7 & 8 & 9 & 10 & 9 & 10 & 11 & 8 & 10 \\
\hline$\%$ & 0.96 & 0.97 & 0.91 & 0.93 & 0.98 & 0.99 & 0.98 & 0.98 & 0.97 & 0.98 & 0.94 \\
\hline
\end{tabular}

TABle 5. $M=100, \alpha=0.05$

\begin{tabular}{|l|lllllllllll|}
\hline$l$ & 4 & 5 & 6 & 8 & 10 & 10 & 10 & 10 & 12 & 13 & 13 \\
$k$ & 2 & 2 & 2 & 2 & 2 & 4 & 6 & 8 & 6 & 6 & 7 \\
\hline$\%$ & 0.21 & 0.23 & 0.14 & 0.22 & 0.25 & 0.53 & 0.90 & 0.99 & 0.84 & 0.88 & 0.93 \\
\hline \hline$l$ & 13 & 14 & 14 & 14 & 14 & 15 & 15 & 15 & 16 & 16 & 16 \\
$k$ & 8 & 7 & 8 & 9 & 10 & 9 & 10 & 11 & 8 & 10 & 12 \\
\hline$\%$ & 0.90 & 0.91 & 0.92 & 0.96 & 0.98 & 0.95 & 0.98 & 0.95 & 0.94 & 0.93 & 0.94 \\
\hline
\end{tabular}

TABLE $6 . \quad M=100, \alpha=0.05, l=12$

\begin{tabular}{|l|llllllll|}
\hline$k$ & 4 & 5 & 6 & 7 & 8 & 9 & 10 & 11 \\
\hline$\%$ & 0.66 & 0.75 & 0.83 & 0.92 & 0.94 & 0.94 & 0.95 & 1 \\
\hline
\end{tabular}

TABLE 7. $M=100, \alpha=0.05, l=14$

\begin{tabular}{|l|llllllllll|}
\hline$k$ & 4 & 5 & 6 & 7 & 8 & 9 & 10 & 11 & 12 & 13 \\
\hline$\%$ & 0.67 & 0.75 & 0.85 & 0.85 & 0.95 & 0.94 & 0.96 & 0.99 & 1 & 1 \\
\hline
\end{tabular}


TABLE 8. $M=100, \alpha=0.05, l=16$

\begin{tabular}{|l|llllll|}
\hline$k$ & 4 & 5 & 6 & 7 & 8 & 9 \\
\hline$\%$ & 0.60 & 0.67 & 0.86 & 0.85 & 0.94 & 0.97 \\
\hline \hline$k$ & 10 & 11 & 12 & 13 & 14 & 15 \\
\hline$\%$ & 0.94 & 0.94 & 0.94 & 0.95 & 0.98 & 0.99 \\
\hline
\end{tabular}

causes increase of percentage, in the sense that percentage, for large enough $k$, is value close to $1-\alpha$, for given $\alpha$, what we expected.

ExAmPLE 6.3. Let us consider ergodic recurrent diffusion $X$ which satisfies SDE (1.1). Let $\bar{\theta}_{n, T}$ and $\hat{\theta}_{T}$ be AMLE and MLE of drift parameters over interval $[0, T]$. Let us assume that

$$
\begin{gathered}
\lim _{T \rightarrow+\infty} \hat{\theta}_{T}=\theta_{0} \text { a.s., } \lim _{T \rightarrow+\infty} \frac{1}{T} D^{2} L_{T}\left(\hat{\theta}_{T}\right)=-M_{0} \text { a.s., } \\
\sqrt{T}\left(\hat{\theta}_{T}-\theta_{0}\right) \Rightarrow N\left(0, M_{0}^{-1}\right), T \rightarrow+\infty, \text { and } \lim _{T \rightarrow+\infty} \frac{1}{T} \Sigma\left(\hat{a}_{T}\right)=\Sigma_{0} \text { a.s. }
\end{gathered}
$$

for some $d \times d$ positive definite matrices $M_{0}$ and $\Sigma_{0}$. Since the difference $\sqrt{T}\left(\bar{\theta}_{n, T}-\theta_{0}\right)$ can be written in the form

$$
\sqrt{T}\left(\bar{\theta}_{n, T}-\theta_{0}\right)=\sqrt{\Delta_{n} T} \frac{\bar{\theta}_{n, T}-\hat{\theta}_{T}}{\sqrt{\Delta_{n}}}+\sqrt{T}\left(\hat{\theta}_{T}-\theta_{0}\right),
$$

for large enough $T$ and $n$ one can say that the difference $\sqrt{T}\left(\bar{\theta}_{n, T}-\theta_{0}\right)$ asymptotically behaves as $\sqrt{\Delta_{n}}\left(\frac{1}{T} D^{2} L_{T}\left(\hat{\theta}_{T}\right)\right)^{-1}\left(\frac{1}{T} \Sigma\left(\hat{\theta}_{T}\right)\right)^{\frac{1}{2}} Z_{T}+\left(M_{0}^{-1}\right)^{\frac{1}{2}} Z$. But if $T$ is large enough, then we can say that the difference $\sqrt{T}\left(\bar{\theta}_{n, T}-\theta_{0}\right)$ asymptotically behaves as $\sqrt{\Delta_{n}}\left(-M_{0}\right)^{-1}\left(\Sigma_{0}\right)^{\frac{1}{2}} Z_{T}+\left(M_{0}^{-1}\right)^{\frac{1}{2}} Z$, where $Z_{T}$ is standard normal random vector independent of $\mathcal{F}_{T}$, and $Z$ is standard normal random vector. For example, if we look at the variance at one dimensional case

$$
\begin{aligned}
\operatorname{Var} & \left.\sqrt{\Delta_{n}}\left(\frac{1}{T} D^{2} L_{T}\left(\hat{\theta}_{T}\right)\right)^{-1}\left(\frac{1}{T} \Sigma\left(\hat{\theta}_{T}\right)\right)^{\frac{1}{2}} Z_{T}+\sqrt{T}\left(\hat{\theta}_{T}-a_{0}\right)\right) \\
& =\operatorname{Var}\left(\sqrt{\Delta_{n}}\left(\frac{1}{T} D^{2} L_{T}\left(\hat{\theta}_{T}\right)\right)^{-1}\left(\frac{1}{T} \Sigma\left(\hat{\theta}_{T}\right)\right)^{\frac{1}{2}} Z_{T}\right)+\operatorname{Var}\left(\sqrt{T}\left(\hat{\theta}_{T}-a_{0}\right)\right),
\end{aligned}
$$

then for simulation study we can use this variance to explain standard error and to get asymptotic confidence intervals for true parameter value. This is only the hint how to use the result presented in this paper in future simulation study in the case of ergodic diffusions. 


\section{APPENDIX}

Proof of Lemma 5.2. For the $j$-th component of $\frac{1}{\sqrt{\Delta_{n}}}\left(D L_{T}(\theta)-\right.$ $\left.D L_{n}(\theta)\right), j=1, \ldots, d$, using Itô's formula, we get

$$
\begin{aligned}
\frac{1}{\sqrt{\Delta_{n}}} & \left(D L_{T}(\theta)-D L_{n}(\theta)\right)_{j} \\
= & \frac{1}{\sqrt{\Delta_{n}}} \sum_{i=1}^{n} \int_{t_{i-1}}^{t_{i}} \frac{\mu\left(X_{s}, \theta_{0}\right)}{\sigma_{0}^{2}}\left(\int_{t_{i-1}}^{s}\left(g_{j}^{\prime} \mu\left(\cdot, \theta_{0}\right)+g_{j}^{\prime \prime} \frac{\sigma_{0}^{2}}{2} \nu^{2}\right)\left(X_{u}\right) d u\right) d s \\
& -\frac{1}{\sqrt{\Delta_{n}}} \sum_{i=1}^{n} \int_{t_{i-1}}^{t_{i}} \frac{1}{\sigma_{0}^{2}}\left(\int_{t_{i-1}}^{s}\left(f_{j}^{\prime} \mu\left(\cdot, \theta_{0}\right)+f_{j}^{\prime \prime} \frac{\sigma_{0}^{2}}{2} \nu^{2}\right)\left(X_{u}\right) d u\right) d s \\
& +\frac{1}{\sqrt{\Delta_{n}}} \sum_{i=1}^{n} \int_{t_{i-1}}^{t_{i}} \frac{\mu\left(X_{s}, \theta_{0}\right)}{\sigma_{0}}\left(\int_{t_{i-1}}^{s} g_{j}^{\prime}\left(X_{u}\right) \nu\left(X_{u}\right) d W_{u}\right) d s \\
& -\frac{1}{\sqrt{\Delta_{n}}} \sum_{i=1}^{n} \int_{t_{i-1}}^{t_{i}} \frac{1}{\sigma_{0}}\left(\int_{t_{i-1}}^{s} f_{j}^{\prime}\left(X_{u}\right) \nu\left(X_{u}\right) d W_{u}\right) d s \\
& +\frac{1}{\sqrt{\Delta_{n}}} \sum_{i=1}^{n} \int_{t_{i-1}}^{t_{i}} \frac{\nu\left(X_{s}\right)}{\sigma_{0}}\left(\int_{t_{i-1}}^{s}\left(g_{j}^{\prime} \mu\left(\cdot, \theta_{0}\right)+g_{j}^{\prime \prime} \frac{\sigma_{0}^{2}}{2} \nu^{2}\right)\left(X_{u}\right) d u\right) d W_{s} \\
& +\frac{1}{\sqrt{\Delta_{n}}} \sum_{i=1}^{n} \int_{t_{i-1}}^{t_{i}} \nu\left(X_{s}\right)\left(\int_{t_{i-1}}^{s} g_{j}^{\prime}\left(X_{u}\right) \nu\left(X_{u}\right) d W_{u}\right) d W_{s} .
\end{aligned}
$$

If we define $Z_{n, j}(\theta):=($ A.1 $)+($ A.2) $+($ A. .3$)+($ A.4 $)+($ A.5 $)$, then

$$
Z_{n}(\theta)=\left[\begin{array}{c}
Z_{n, 1}(\theta) \\
\vdots \\
Z_{n, d}(\theta)
\end{array}\right] .
$$

If, for some $j=1, \ldots, d, g_{j}$ is a constant function, then we define $Z_{n, j}(\theta)$ as $Z_{n, j}(\theta):=($ A.2 $)+\left(\right.$ A.4). If, for some $j=1, \ldots, d, f_{j}$ is a constant function, then we define $Z_{n, j}(\theta)$ as $Z_{n, j}(\theta):=($ A.1) + (A.3) + (A.5) + (A.6). So, the conclusion and the proof of this Lemma stay the same in those special cases. It is sufficient to prove that each component $Z_{n, j}(\theta), j=1, \ldots, d$, of the vector $Z_{n}(\theta)$ converges in probability to zero. Using (A6) it is easy to prove that there exists constant $k_{1}>0$, such that

$$
\begin{aligned}
& \mid \frac{1}{\sqrt{\Delta_{n}}} \sum_{i=1}^{n} \int_{t_{i-1}}^{t_{i}} \frac{\mu\left(X_{s}, \theta_{0}\right)}{\sigma_{0}^{2}}\left(\int_{t_{i-1}}^{s}\left(g_{j}^{\prime}\left(X_{u}\right) \mu\left(X_{u}, \theta_{0}\right)+\frac{1}{2} g_{j}^{\prime \prime}\left(X_{u}\right) \sigma_{0}^{2} \nu^{2}\left(X_{u}\right)\right) d u\right) d s \\
& -\frac{1}{\sqrt{\Delta_{n}}} \sum_{i=1}^{n} \int_{t_{i-1}}^{t_{i}} \frac{1}{\sigma_{0}^{2}}\left(\int_{t_{i-1}}^{s}\left(f_{j}^{\prime}\left(X_{u}\right) \mu\left(X_{u}, \theta_{0}\right)+\frac{1}{2} f_{j}^{\prime \prime}\left(X_{u}\right) \sigma_{0}^{2} \nu^{2}\left(X_{u}\right)\right) d u\right) d s \mid \\
& \quad \leq k_{1} \sqrt{\Delta_{n}} T,
\end{aligned}
$$


so we conclude that $(\mathrm{A} .1)+(\mathrm{A} .2)$ converges in probability to zero.

Let us denote with $\mu^{\prime}$ and $\mu^{\prime \prime}$ first and second derivative of the function $x \mapsto \mu\left(x, \theta_{0}\right)$ with respect to $x$. For (A.3), we have

$$
\begin{aligned}
\frac{1}{\sqrt{\Delta_{n}}} & \sum_{i=1}^{n} \int_{t_{i-1}}^{t_{i}} \frac{\mu\left(X_{s}, \theta_{0}\right)}{\sigma_{0}}\left(\int_{t_{i-1}}^{s} g_{j}^{\prime}\left(X_{u}\right) \nu\left(X_{u}\right) d W_{u}\right) d s \\
\text { A.7 })= & \frac{1}{\sqrt{\Delta_{n}}} \sum_{i=1}^{n} \int_{t_{i-1}}^{t_{i}} \int_{t_{i-1}}^{s} \frac{\mu\left(X_{i-1}, \theta_{0}\right)}{\sigma_{0}} g_{j}^{\prime}\left(X_{u}\right) \nu\left(X_{u}\right) d W_{u} d s \\
& +\frac{1}{\sqrt{\Delta_{n}}} \sum_{i=1}^{n} \int_{t_{i-1}}^{t_{i}}\left(\int_{t_{i-1}}^{s}\left(\mu^{\prime}\left(\cdot, \theta_{0}\right) \mu\left(\cdot, \theta_{0}\right)+\frac{1}{2} \mu^{\prime \prime}\left(\cdot, \theta_{0}\right) \sigma_{0}^{2} \nu^{2}\right)\left(X_{u}\right) d u\right) .
\end{aligned}
$$

$$
\begin{aligned}
& \cdot\left(\int_{t_{i-1}}^{s} \frac{1}{\sigma_{0}} g_{j}^{\prime}\left(X_{u}\right) \nu\left(X_{u}\right) d W_{u}\right) d s \\
+ & \left.\frac{1}{\sqrt{\Delta_{n}}} \sum_{i=1}^{n} \int_{t_{i-1}}^{t_{i}} \int_{t_{i-1}}^{s}\left(\int_{t_{i-1}}^{u}\left(\mu^{\prime}\left(\cdot, \theta_{0}\right) \nu\right)\left(X_{l}\right) d W_{l}\right) \cdot\left(g_{j}^{\prime} \nu\right)\left(X_{u}\right)\right) d W_{u} d s \\
+ & \frac{1}{\sqrt{\Delta_{n}}} \sum_{i=1}^{n} \int_{t_{i-1}}^{t_{i}} \int_{t_{i-1}}^{s}\left(\int_{t_{i-1}}^{u}\left(g_{j}^{\prime} \nu\right)\left(X_{l}\right) d W_{l}\right) \cdot\left(\mu^{\prime}\left(\cdot, \theta_{0}\right) \nu\right)\left(X_{u}\right) d W_{u} d s \\
+ & \frac{1}{\sqrt{\Delta_{n}}} \sum_{i=1}^{n} \int_{t_{i-1}}^{t_{i}} \int_{t_{i-1}}^{s} \mu^{\prime}\left(X_{u}, \theta_{0}\right) \nu^{2}\left(X_{u}\right) g_{j}^{\prime}\left(X_{u}\right) d u d s,
\end{aligned}
$$

where (A.9)-(A.11) we get by applying Itô's formula for

$$
\int_{t_{i-1}}^{s} \mu^{\prime}\left(X_{u}, \theta_{0}\right) \nu\left(X_{u}\right) d W_{u} \int_{t_{i-1}}^{s} g_{j}^{\prime}\left(X_{u}\right) \nu\left(X_{u}\right) d W_{u} .
$$

In the case when $\mu\left(\cdot, \theta_{0}\right)$ is a constant function, (A.3) would have the same form as (A.7). It can be shown that there exist constants $k_{2}, k_{3}, k_{4}, k_{5}, k_{6}$ such that

$$
\begin{aligned}
& \left\|\frac{1}{\sqrt{\Delta_{n}}} \sum_{i=1}^{n} \int_{t_{i-1}}^{t_{i}} \int_{t_{i-1}}^{s} \frac{\mu\left(X_{i-1}, \theta_{0}\right)}{\sigma_{0}} g_{j}^{\prime}\left(X_{u}\right) \nu\left(X_{u}\right) d W_{u} d s\right\|_{2} \leq k_{2} \sqrt{\Delta_{n}} \sqrt{T}, \\
& \| \frac{1}{\sqrt{\Delta_{n}}} \sum_{i=1}^{n} \int_{t_{i-1}}^{t_{i}}\left(\int_{t_{i-1}}^{s}\left(\mu^{\prime}\left(X_{u}, \theta_{0}\right) \mu\left(X_{u}, \theta_{0}\right)+\frac{1}{2} \mu^{\prime \prime}\left(X_{u}, \theta_{0}\right) \sigma_{0}^{2} \nu^{2}\left(X_{u}\right)\right) d u\right) . \\
& \quad \cdot\left(\int_{t_{i-1}}^{s} \frac{1}{\sigma_{0}} g_{j}^{\prime}\left(X_{u}\right) \nu\left(X_{u}\right) d W_{u}\right) d s \|_{2} \\
& \leq k_{3} \sqrt{\Delta_{n}} T \\
& \quad\left\|\frac{1}{\sqrt{\Delta_{n}}} \sum_{i=1}^{n} \int_{t_{i-1}}^{t_{i}} \int_{t_{i-1}}^{s}\left(\int_{t_{i-1}}^{u} \mu^{\prime}\left(X_{l}, \theta_{0}\right) \nu\left(X_{l}\right) d W_{l}\right) \cdot\left(g_{j}^{\prime}\left(X_{u}\right) \nu\left(X_{u}\right)\right) d W_{u} d s\right\|_{2} \\
& \leq k_{4} \Delta_{n} \sqrt{T}
\end{aligned}
$$




$$
\begin{aligned}
& \left\|\frac{1}{\sqrt{\Delta_{n}}} \sum_{i=1}^{n} \int_{t_{i-1}}^{t_{i}} \int_{t_{i-1}}^{s}\left(\int_{t_{i-1}}^{u} g_{j}^{\prime}\left(X_{l}\right) \nu\left(X_{l}\right) d W_{l}\right) \cdot\left(\mu^{\prime}\left(X_{u}, \theta_{0}\right) \nu\left(X_{u}\right)\right) d W_{u} d s\right\|_{2} \\
\leq & k_{5} \Delta_{n} \sqrt{T} \\
& \left|\frac{1}{\sqrt{\Delta_{n}}} \sum_{i=1}^{n} \int_{t_{i-1}}^{t_{i}} \int_{t_{i-1}}^{s} \mu^{\prime}\left(X_{u}, \theta_{0}\right) \nu^{2}\left(X_{u}\right) g_{j}^{\prime}\left(X_{u}\right) d u d s\right| \leq k_{6} \sqrt{\Delta_{n}} T,
\end{aligned}
$$

so (A.7),(A.8),(A.9) and (A.10) converge in $L^{2}$-norm to zero, and (A.11) converges almost surely to zero. From this we conclude that (A.3) converges in probability to zero.

For example, let us show how we can get constant $k_{2}$. The other constants in this article can be calculated in similar way. For (A.7) we have

$$
\begin{aligned}
\mathbb{E}[( & \left.\left.\frac{1}{\sqrt{\Delta_{n}}} \sum_{i=1}^{n} \int_{t_{i-1}}^{t_{i}} \int_{t_{i-1}}^{s} \frac{\mu\left(X_{i-1}, \theta_{0}\right)}{\sigma_{0}} g_{j}^{\prime}\left(X_{u}\right) \nu\left(X_{u}\right) d W_{u} d s\right)^{2}\right] \\
= & \frac{1}{\Delta_{n}} \sum_{i=1}^{n} \mathbb{E}\left[\left(\int_{t_{i-1}}^{t_{i}} \int_{t_{i-1}}^{s} \frac{\mu\left(X_{i-1}, \theta_{0}\right)}{\sigma_{0}} g_{j}^{\prime}\left(X_{u}\right) \nu\left(X_{u}\right) d W_{u} d s\right)^{2}\right] \\
& +\frac{2}{\Delta_{n}} \sum_{1 \leq i<k \leq n} \mathbb{E}\left[\left(\int_{t_{i-1}}^{t_{i}} \int_{t_{i-1}}^{s} \frac{\mu\left(X_{i-1}, \theta_{0}\right)}{\sigma_{0}} g_{j}^{\prime}\left(X_{u}\right) \nu\left(X_{u}\right) d W_{u} d s\right) .\right. \\
= & \frac{1}{\Delta_{n}} \sum_{i=1}^{n} \mathbb{E}\left[\left(\int_{t_{i-1}}^{t_{i}} \int_{t_{i-1}}^{s} \frac{\mu\left(X_{i-1}, \theta_{0}\right)}{\sigma_{0}} g_{j}^{\prime}\left(X_{u}\right) \nu\left(X_{u}\right) d W_{u} d s\right)^{2}\right] \\
\leq & \frac{1}{\Delta_{n}} \sum_{i=1}^{n} \Delta_{n} \int_{t_{i-1}}^{t_{i}} \mathbb{E}\left[\left(\int_{t_{i-1}}^{s} \frac{\mu\left(X_{i-1}, \theta_{0}\right)}{\sigma_{0}} g_{j}^{\prime}\left(X_{u}\right) \nu\left(X_{u}\right) d W_{u}\right)^{2}\right] d s \\
= & \frac{1}{\Delta_{n}} \sum_{i=1}^{n} \Delta_{n} \int_{t_{i-1}}^{t_{i}} \int_{t_{i-1}}^{s} \mathbb{E}\left[\left(\frac{\mu\left(X_{i-1}, \theta_{0}\right)}{\sigma_{0}} g_{j}^{\prime}\left(X_{u}\right) \nu\left(X_{u}\right)\right)^{2}\right] d u d s \\
\leq & \frac{k_{2}^{2}}{\Delta_{n}} \sum_{i=1}^{n} \Delta_{n}^{3} \leq k_{2}^{2} \Delta_{n} T .
\end{aligned}
$$

For (A.4) there exists constant $k_{7}$ such that

$$
\left\|\frac{1}{\sqrt{\Delta_{n}}} \sum_{i=1}^{n} \int_{t_{i-1}}^{t_{i}} \frac{1}{\sigma_{0}}\left(\int_{t_{i-1}}^{s} f_{j}^{\prime}\left(X_{u}\right) \nu\left(X_{u}\right) d W_{u}\right) d s\right\|_{2} \leq k_{7} \sqrt{\Delta_{n}} \sqrt{T}
$$


from which follows that (A.4) converges in probability to zero. For (A.5) there exists constant $k_{8}$ such that

$$
\begin{aligned}
\| \frac{1}{\sqrt{\Delta_{n}}} & \sum_{i=1}^{n} \int_{t_{i-1}}^{t_{i}} \frac{\nu\left(X_{s}\right)}{\sigma_{0}}\left(\int_{t_{i-1}}^{s}\left(g_{j}^{\prime}\left(X_{u}\right) \mu\left(X_{u}, \theta_{0}\right)+\frac{1}{2} g_{j}^{\prime \prime}\left(X_{u}\right) \sigma_{0}^{2} \nu^{2}\left(X_{u}\right)\right) d u\right) d W_{s} \|_{2} \\
& \leq k_{8} \sqrt{\Delta_{n}} \sqrt{T},
\end{aligned}
$$

from which we conclude that (A.5) converges in probability to zero. We can conclude that $Z_{n}(\theta)$ converges in probability to zero.

Proof of Lemma 5.7. Since

$$
\begin{aligned}
& \left\{\sup _{v \in[0,1]}\left|\left(D^{2} L_{T}(\hat{\theta})-D^{2} L_{T}\left(\hat{\theta}+v\left(\bar{\theta}_{n}-\hat{\theta}\right)\right)\right)_{i j}\right|<\epsilon\right\} \\
& \quad \subseteq\left\{\left|\int_{0}^{1}\left(D^{2} L_{T}(\hat{\theta})-D^{2} L_{T}\left(\hat{\theta}+v\left(\bar{\theta}_{n}-\hat{\theta}\right)\right)\right)_{i j} d v\right|<\epsilon\right\},
\end{aligned}
$$

for $i, j=1, \ldots, d$ it is sufficient to prove that

$$
\sup _{v \in[0,1]}\left\|D^{2} L_{T}(\hat{\theta})-D^{2} L_{T}\left(\hat{\theta}+v\left(\bar{\theta}_{n}-\hat{\theta}\right)\right)\right\| \stackrel{\mathbb{P}}{\rightarrow} 0 .
$$

Let us define the functions

$$
F(\theta, v) \equiv F(\omega, \theta, v):=\left\|D^{2} L_{T}(\omega, \hat{\theta}(\omega))-D^{2} L_{T}(\omega, \hat{\theta}(\omega)+v(\theta-\hat{\theta}(\omega)))\right\|
$$

Function $F$ is uniformly continuous on $C l(\Theta) \times[0,1]$, for each $\omega \in \Omega$ and the functions $\sup _{v \in[0,1]} F(\theta, v)$ are continuous for each $\omega \in \Omega$.

We want to prove that

$$
\sup _{v \in[0,1]} F\left(\bar{\theta}_{n}, v\right) \stackrel{\mathbb{P}}{\rightarrow} 0 .
$$

If it is not true then there exist $\epsilon, \delta>0$ such that for all $k \in \mathbb{N}$ exists $n_{k} \geq k$ such that

$$
\mathbb{P}\left(\omega:\left|\sup _{v \in[0,1]} F\left(\omega, \bar{\theta}_{n_{k}}(\omega), v\right)\right| \geq \epsilon\right) \geq \delta
$$

(4.3) implies $\bar{\theta}_{n_{k}} \stackrel{\mathbb{P}}{\rightarrow} \hat{\theta_{T}}$, when $k \rightarrow \infty$, hence there exist subsequence $\left(\bar{\theta}_{n_{k_{j}}}\right)$ of the sequence $\left(\bar{\theta}_{n_{k}}\right)$ such that

$$
\bar{\theta}_{n_{k_{j}}} \stackrel{a . s .}{\rightarrow} \hat{\theta}, \quad j \rightarrow \infty .
$$

Let us denote by $\Omega_{0}$ the event such that $\mathbb{P}\left(\Omega_{0}\right)=1$ and for all $\omega \in \Omega_{0}$ holds $\bar{\theta}_{n_{k_{j}}}(\omega) \rightarrow \hat{\theta}(\omega), j \rightarrow \infty$. Let $\omega \in \Omega_{0}$. Then, because of continuity of the function $\sup _{v \in[0,1]} F(\omega, \cdot, v)$, follows

$$
\sup _{v \in[0,1]} F\left(\omega, \bar{\theta}_{n_{k_{j}}}(\omega), v\right) \rightarrow \sup _{v \in[0,1]} F(\omega, \hat{\theta}(\omega), v)=0, \quad j \rightarrow \infty,
$$


from which we conclude that $\sup _{v \in[0,1]} F\left(\bar{\theta}_{n_{k_{j}}}, v\right)$ converges almost surely to 0 , when $j \rightarrow \infty$, hence it converges in probability to 0 , which is contradiction with (A.12).

Proof of Lemma 5.8. For the sequence $D^{2} L_{T}(\hat{\theta}) \frac{1}{\sqrt{\Delta_{n}}}\left(\bar{\theta}_{n}-\hat{\theta}\right)$ holds

$$
\begin{aligned}
D^{2} L_{T}(\hat{\theta}) \frac{1}{\sqrt{\Delta_{n}}}\left(\bar{\theta}_{n}-\hat{\theta}\right) \\
=\int_{0}^{1}\left(D^{2} L_{T}(\hat{\theta})-D^{2} L_{T}\left(\hat{\theta}+v\left(\bar{\theta}_{n}-\hat{\theta}\right)\right)\right) d v \frac{1}{\sqrt{\Delta_{n}}}\left(\bar{\theta}_{n}-\hat{\theta}\right) \\
\quad+\int_{0}^{1}\left(D^{2} L_{T}\left(\hat{\theta}+v\left(\bar{\theta}_{n}-\hat{\theta}\right)\right)-D^{2} L_{n}\left(\hat{\theta}+v\left(\bar{\theta}_{n}-\hat{\theta}\right)\right)\right) d v \frac{1}{\sqrt{\Delta_{n}}}\left(\bar{\theta}_{n}-\hat{\theta}\right) \\
\quad+A_{\hat{\theta}, \bar{\theta}_{n}} \frac{1}{\sqrt{\Delta_{n}}}\left(\bar{\theta}_{n}-\hat{\theta}\right) .
\end{aligned}
$$

Sequence $\frac{1}{\sqrt{\Delta_{n}}}\left(\bar{\theta}_{n}-\hat{\theta}\right)$ is bounded in probability, so by Lemma 5.7 follows

$$
\int_{0}^{1}\left(D^{2} L_{T}(\hat{\theta})-D^{2} L_{T}\left(\hat{\theta}+v\left(\bar{\theta}_{n}-\hat{\theta}\right)\right)\right) d v \frac{1}{\sqrt{\Delta_{n}}}\left(\bar{\theta}_{n}-\hat{\theta}\right) \stackrel{\mathbb{P}}{\rightarrow} 0 .
$$

(4.6) yields $\sup _{v \in[0,1]} \|\left(D^{2} L_{T}\left(\hat{\theta}+v\left(\bar{\theta}_{n}-\hat{\theta}\right)\right)-D^{2} L_{n}\left(\hat{\theta}+v\left(\bar{\theta}_{n}-\hat{\theta}\right)\right) \| \stackrel{\mathbb{P}}{\rightarrow} 0\right.$. Hence, we can conclude that

$$
\int_{0}^{1}\left(D^{2} L_{T}\left(\hat{\theta}+v\left(\bar{\theta}_{n}-\hat{\theta}\right)\right)-D^{2} L_{n}\left(\hat{\theta}+v\left(\bar{\theta}_{n}-\hat{\theta}\right)\right)\right) d v \stackrel{\mathbb{P}}{\rightarrow} 0
$$

which implies

$$
\int_{0}^{1}\left(D^{2} L_{T}\left(\hat{\theta}+v\left(\bar{\theta}_{n}-\hat{\theta}\right)\right)-D^{2} L_{n}\left(\hat{\theta}+v\left(\bar{\theta}_{n}-\hat{\theta}\right)\right)\right) d v \frac{1}{\sqrt{\Delta_{n}}}\left(\bar{\theta}_{n}-\hat{\theta}\right) \stackrel{\mathbb{P}}{\rightarrow} 0 .
$$

Lemma 5.6 and $[11,(2.2)]$ yields the conclusion.

Proof OF Lemma 5.11. It is sufficient to prove that Lemma holds for each $j k$ component of matrices. Since

$$
\Sigma_{n}\left(\bar{\theta}_{n}\right)^{j k}=\Sigma_{n}\left(\bar{\theta}_{n}\right)^{j k}-\Sigma\left(\bar{\theta}_{n}\right)^{j k}+\Sigma\left(\bar{\theta}_{n}\right)^{j k}-\Sigma(\hat{\theta})^{j k}+\Sigma(\hat{\theta})^{j k},
$$

it is sufficient to prove that $\Sigma_{n}\left(\bar{\theta}_{n}\right)^{j k}-\Sigma\left(\bar{\theta}_{n}\right)^{j k} \stackrel{\mathbb{P}}{\rightarrow} 0$ and $\Sigma\left(\bar{\theta}_{n}\right)^{j k}-\Sigma(\hat{\theta})^{j k} \stackrel{\mathbb{P}}{\rightarrow}$ 0 . Let $\omega \in \Omega$ be fixed. Then the functions $\bar{f}_{j k}:[0, T] \rightarrow \mathbb{R}$, defined by $\bar{f}_{j k}(t):=f_{j k}\left(X_{t}(\omega), \bar{\theta}_{n}(\omega)\right)$, are uniformly continuous. Let $\epsilon>0$ be arbitrary fixed number. Then, there exists $\delta>0$, such that

$$
|s-t|<\delta \Rightarrow\left|f_{j k}\left(X_{s}, \bar{\theta}_{n}\right)-f_{j k}\left(X_{t}, \bar{\theta}_{n}\right)\right|<\frac{\epsilon}{T} .
$$


Let $n_{0} \in \mathbb{N}$, such that for all $n \geq n_{0}$ holds $\Delta_{n}<\delta$. Then

$$
\begin{aligned}
\left|\Sigma_{n}\left(\bar{\theta}_{n}\right)^{j k}-\Sigma\left(\bar{\theta}_{n}\right)^{j k}\right| & =\sum_{i=1}^{n} \int_{t_{i-1}}^{t_{i}}\left(f_{j k}\left(X_{t_{i-1}}, \bar{\theta}_{n}\right)-f_{j k}\left(X_{s}, \bar{\theta}_{n}\right)\right) d s \\
& <\sum_{i=1}^{n} \frac{\epsilon}{T} \Delta_{n}=\epsilon
\end{aligned}
$$

so $\Sigma_{n}\left(\bar{\theta}_{n}\right)^{j k}-\Sigma\left(\bar{\theta}_{n}\right)^{j k} \stackrel{a . s .}{\rightarrow} 0$, which implies $\Sigma_{n}\left(\bar{\theta}_{n}\right)^{j k}-\Sigma\left(\bar{\theta}_{n}\right)^{j k} \stackrel{\mathbb{P}}{\rightarrow} 0$. For fixed $\omega \in \Omega$ and $s \in[0, T]$ let $\hat{f}_{j k, s}: \Theta \rightarrow \mathbb{R}$ be the function defined by $\hat{f}_{j k, s}(\theta)=f_{j k}\left(X_{s}(\omega), \theta\right)$. (A3) yields that $\hat{f}_{j k, s} \in C^{1}(\Theta)$, for all $\omega \in \Omega$, hence

$$
\left|\hat{f}_{j k, s}\left(\bar{\theta}_{n}\right)-\hat{f}_{j k, s}(\hat{\theta})\right| \leq \sum_{l=1}^{d} \sup _{\theta \in \Theta}\left|\partial \theta_{l} \hat{f}_{j k, s}(\theta)\right|\left\|\bar{\theta}_{n}-\hat{\theta}\right\| .
$$

If we assume that all of the functions are bounded, then there exists constant $M>0$ such that

$$
\left|\hat{f}_{j k, s}\left(\bar{\theta}_{n}\right)-\hat{f}_{j k, s}(\hat{\theta})\right| \leq M\left\|\bar{\theta}_{n}-\hat{\theta}\right\| .
$$

Let $\epsilon>0$ be arbitrary, fixed number. Let $\delta>0$. Then, due to (4.3), there exists $n_{0} \in \mathbb{N}$ such that for all $n \geq n_{0}$ holds

$$
\mathbb{P}\left(\left\|\bar{\theta}_{n}-\hat{\theta}\right\| \geq \frac{\delta}{M T}\right)<\epsilon
$$

For $n \geq n_{0}$ let us define the set $E_{n}:=\left\{\left\|\bar{\theta}_{n}-\hat{\theta}\right\|<\frac{\delta}{M T}\right\}$. Let $\omega \in E_{n}$. Then

$$
\left|\hat{f}_{j k, s}\left(\bar{\theta}_{n}\right)-\hat{f}_{j k, s}(\hat{\theta})\right|=\left|\left(f_{j k}\left(X_{s}, \bar{\theta}_{n}\right)-f_{j k}\left(X_{s}, \hat{\theta}\right)\right)\right|<M \frac{\delta}{M T}=\frac{\delta}{T},
$$

for all $s \in[0, T]$, which implies

$$
\left|\int_{0}^{T}\left(f_{j k}\left(X_{s}, \bar{\theta}_{n}\right)-f_{j k}\left(X_{s}, \hat{\theta}\right)\right) d s\right|<\delta,
$$

hence

$$
\mathbb{P}\left(\left|\int_{0}^{T}\left(f_{j k}\left(X_{s}, \bar{\theta}_{n}\right)-f_{j k}\left(X_{s}, \hat{\theta}\right)\right) d s\right| \geq \delta\right) \leq \mathbb{P}\left(E_{n}^{c}\right)<\epsilon,
$$

which proves that $\Sigma\left(\bar{\theta}_{n}\right)^{j k}-\Sigma(\hat{\theta})^{j k} \stackrel{\mathbb{P}}{\rightarrow} 0$. For general case convergence in probability can be proved by defining the stopping times $T_{M}$, as seen before.

Proof of Lemma 5.12. Let $\epsilon>0$ such that $\lambda_{\Sigma}-\epsilon>0$. Lemma 5.11 implies $\lambda_{1}\left(\Sigma_{n}\left(\bar{\theta}_{n}\right)\right)-\lambda_{1}(\Sigma(\hat{\theta})) \stackrel{\mathbb{P}}{\rightarrow} 0$. Since $\Sigma_{n}\left(\bar{\theta}_{n}\right)$ is symmetric real matrix, for all $\omega \in \Omega$, it follows that

$$
\mathbb{P}\left(\Sigma_{n}\left(\bar{\theta}_{n}\right) \text { is regular matrix }\right)=\mathbb{P}\left(\lambda_{1}\left(\Sigma_{n}\left(\bar{\theta}_{n}\right)\right)>0\right) .
$$


Since

$\left\{\left|\lambda_{1}\left(\Sigma_{n}\left(\bar{\theta}_{n}\right)\right)-\lambda_{1}(\Sigma(\hat{\theta}))\right|<\epsilon\right\} \subseteq\left\{\lambda_{\Sigma}-\epsilon<\lambda_{1}\left(\Sigma_{n}\left(\bar{\theta}_{n}\right)\right)\right\} \subseteq\left\{0<\lambda_{1}\left(\Sigma_{n}\left(\bar{\theta}_{n}\right)\right)\right\}$,

we can conclude that $\mathbb{P}\left(\lambda_{1}\left(\Sigma_{n}\left(\bar{\theta}_{n}\right)\right)>0\right) \stackrel{\mathbb{P}}{\rightarrow} 1$, which proves our Lemma.

ACKNOWLEDGEMENTS.

The authors would like to thank Professor Jean Jacod for many helpful comments and suggestions.

\section{REFERENCES}

[1] D. J. Aldous and G. K. Eagleson, On mixing and stability of limit theorems, Ann. Probability 6 (1978) 325-331.

[2] P. Billingsley, Convergence of probability measures, 2nd edn. Wiley and Sons, New York, 1999

[3] B. M. Brown and J. I. Hewitt, Asymptotic likelihood theory for diffusion processes, J. Appl. Probab. 12 (1975), 228-238.

[4] D. Dacunha-Castelle and D. Florens-Zmirou, Estimation of the coefficients of a diffusion from discrete observations, Stochastics 19 (1986), 263-284.

[5] P. D. Feigin, Maximum likelihood estimation for continuous-time stochastic processes Adv. in Appl. Probab. 8 (1976), 712-736.

[6] D. Florens-Zmirou, Approximate discrete time shemes for statistics of diffusion processes, Statistics 20 (1989), 547-557.

[7] M. Huzak, Selection of diffusion growth process and parameter estimation from discrete observation, Ph.D. thesis, University of Zagreb, (in Croatian), 1997.

[8] M. Huzak, Parameter estimation of diffusion models, Math. Commun. 3 (1998), 129134.

[9] M. Huzak, A general theorem on approximate maximum likelihood estimation, Glas. Mat. Ser. III 36(56) (2001), 139-153.

[10] M. Huzak, Estimating a class of diffusions from discrete observations via approximate maximum likelihood method, Statistics, to appear.

[11] J. Jacod, On continuous conditional Gaussian martingales and stable convergence in law, in: Séminaire de probabilitiés (Strasbourg), XXXI, Lecture Notes in Math. 1655, Springer, Berlin, 1997, 232-246.

[12] J. Jacod and P. Protter, Discretization of processes, Springer, Heidelberg, 2012.

[13] J. Jacod and A. N. Shiryaev, Limit theorems for stochastic processes, Springer-Verlag, Berlin, 2003.

[14] M. Kessler, Estimation of an Ergodic Diffusion from Discrete Observations, Scand. J. Statist. 24 (1997), 211-229.

[15] A. LeBreton, On continuous and discrete sampling for parameter estimation in diffusion type processes, Math. Programming Stud. 5 (1976), 124-144.

[16] R. S. Liptser and A. N. Shiryaev, Statistics of random processes II. Applications, Springer-Verlag, New York-Heidelberg, 1978.

[17] P. Protter (2004). Stochastic integration and differential equations, Springer-Verlag, Berlin, 2004.

[18] A. Rényi, On stable sequences of events, Sankhya Ser. A 25 (1963), 293-302.

[19] D. Revuz and M. Yor, Continuous martingales and Brownian motion, Springer-Verlag, Berlin, 1999.

[20] L. C. G. Rogers and D. Williams, Diffusion, Markov processes, and martingales, Vol. 2. Itô calculus. John Wiley \& Sons, Ltd., New York, 1987. 
S. Lubura Strunjak

Department of Mathematics

Faculty of Science

University of Zagreb

Bijenička 30, 10000 Zagreb

Croatia

E-mail: snjezana.lubura.strunjak@math.hr

M. Huzak

Department of Mathematics

Faculty of Science

University of Zagreb

Bijenička 30, 10000 Zagreb

Croatia

E-mail: miljenko.huzak@math.hr

Received: 9.1.2017.

Revised: 27.6.2017. 